\title{
Analysis of the Generalized DF-CF for Gaussian Relay Channels: Decode or Compress?
}

\author{
Kevin Luo, Ramy H. Gohary, Member, IEEE, and Halim Yanikomeroglu, Senior Member, IEEE
}

\begin{abstract}
We consider a three-node quasi-static communication system with a full-duplex relay. The goal is to determine the relaying mode that enables rate-efficient communication under given channel conditions. To achieve this goal, we consider a generalized scheme that subsumes the decode-and-forward (DF) and compress-and-forward $(C F)$ schemes as special cases. The generalized scheme is considered when the source and relay signals are synthesized from commonly-used Gaussian codebooks, which are shown to be capacity achieving in two asymptotic cases: perfect relay-destination link and broken source-destination link. Studying the generalized DF-CF scheme, it is shown that, for two non-asymptotic cases in which the signal-to-noise ratios (SNRs) of the links satisfy certain conditions, this scheme reduces to either DF or CF. For another set of non-asymptotic SNRs, the generalized scheme is shown to yield strictly higher rates than both DF and CF. Despite the complexity of the generalized scheme, its rate advantage over DF and CF is shown to be upper bounded by 0.5 bits per channel use. This indicates that the practical benefit of the analysis of this scheme is to enable selecting the relaying mode that suits a given channel realization. Numerical results show that, under Rayleigh fading conditions, this selection yields significant gains over fixed DF and CF.
\end{abstract}

Index Terms-Cooperative communication, Full-duplex, KKT conditions.

\section{INTRODUCTION}

$\mathbf{F}$ UTURE advances in wireless communications are expected to bank on the substantial gains provided by effective cooperation between multiple nodes in the network [1]. In its simplest form, cooperation is established when a single relay assists communication between a source-destination pair [2], [3]. Relay operation modes can be categorized into either full-duplex mode, in which the relay uses the same physical channel for transmission and reception, and halfduplex mode, in which these channels are orthogonal [4]. Due to its relative simplicity, half-duplex relaying is better understood than its full-duplex counterpart. However, fullduplex relays are generally more efficient and can enable higher data rates to be reliably communicated. Among the various relaying schemes are decode-and-forward (DF) and compress-and-forward (CF) [5]. In DF, the relay decodes

Manuscript received August 15, 2012; revised December 14, 2012. The associate editor coordinating the review of this paper and approving it for publication was G. Bauch.

The authors are with the Dept. of Systems and Computer Engineering, Carleton University, Ottawa, ON K1S 5B6, Canada.

This work is supported in part by BlackBerry (formerly Research In Motion (RIM)), and in part by the Collaborative Research and Development (CRD) grant of the Natural Sciences and Engineering Research Council (NSERC) of Canada.

A preliminary version of this work appears in the Proc. 2011 IEEE Inf. Theory Wkshp. (ITW).

Digital Object Identifier 10.1109/TCOMM.2013.032013.120601 its observed signal and generates auxiliary information that assists decoding at the destination. In contrast, in CF the relay does not decode its observed signal, but compresses it to generate auxiliary information that facilitates decoding at the destination [5]. When the relay channel is Gaussian, the optimal distribution of the DF signals is Gaussian, whereas the optimal distribution of the CF signals is not known [6], except for the asymptotic case in which the relay-destination link is perfect. In this case, the distribution of the CF codebooks is also Gaussian [7]. Despite the potential rate loss incurred by using Gaussian signals with CF relaying in non-asymptotic cases, at which the SNRs of the links assume finite values, the asymptotic optimality and simplicity of these codebooks have made them commonly used for studying CF relaying in Gaussian channels [6].

Performance analyses of the DF and CF schemes with Gaussian signals were conducted in [6] for special classes of relay channels. For the case of low-power relays and fading channels, an analysis of the rates achieved by DF and time-division multiplexing was conducted in [8]. For those cases, performance comparisons between the DF scheme with direct transmission and other relaying schemes were considered in [9]. It was shown that, for Gaussian relay channels and Gaussian signals, the gap between the rate achieved by the CF scheme and capacity is upper bounded by a constant value when the noise processes at the relay and the destination are independent [10], [11]. In [12], a similar bound was obtained for Gaussian channels with correlated noises. Practical implementation of DF and a variant of CF in halfduplex relay systems was considered in [13] and [14]. In both schemes the relaying mode is chosen depending on successful decoding at the relay. In [13], the performance of the scheme proposed therein is analyzed when decoding failures at the destination are fed back, triggering retransmission. In contrast, the performance analysis of the relaying scheme in [14] does not consider feedback or retransmission. Extensions of the DF and CF schemes are considered in [15] for Gaussian channels with correlated relay and destination noises and in [6], [16] for scalar Rayleigh fading channels. Extensions to multiple antenna channels are considered in [17] and to networks with one source, one destination and multiple relays in [18].

Although DF and CF can achieve the capacity of specific relay channel models [5], [7], [19]-[22], whether these schemes are capacity achieving in other cases is not known. Since the capacity of general relay channels, even with a single relay and simple scalar channels, remains an open problem, it is important to investigate relaying schemes that offer the potential of achieving rates higher than those achieved by 
DF and CF. One such scheme is the so-called quantizemap-forward. This scheme was proposed in [10] and [23] for Gaussian relay networks and was shown to achieve a rate within a constant gap to capacity. Another scheme that might offer higher rates than conventional DF and $\mathrm{CF}$ is the generalized DF-CF scheme which was originally proposed in [5, Theorem 7] and is the focus of the current paper.

The key idea of the generalized DF-CF scheme is to combine DF and CF in one relaying scheme that subsumes both schemes as special cases. In this generalization the relay performs partial decoding and compression of its received signal to generate auxiliary information to assist decoding at the destination. To some extent, the philosophy underlying the generalized DF-CF scheme resembles that of the DF-CF switching proposed in [14] when decoding fails. However, unlike [14] it avoids unsuccessful decoding at the relay and provides deterministic thresholds beyond which the relay ought to switch its operating mode.

The generalized DF-CF scheme has received significantly less attention than either DF or CF. An instance in which a special case of this scheme was used to develop a lower bound on the capacity of the static relay channel was considered in [7]. Another instance was considered in [24], wherein a particular geometric setup of a Gaussian relay network was considered and a numerical demonstration of the rate advantage of the generalized DF-CF scheme over DF and CF was provided. Building on the generalized DF-CF scheme, other potentially more advantageous, mixed DF and CF schemes have been recently proposed in [25]-[27]. Similar to the generalization proposed in [5, Theorem 7], the mixed schemes proposed in [25]-[27] rely on the fundamental mechanism of superimposing DF and CF. Hence, the analysis methodology proposed herein for the generalized DF-CF scheme in [5, Theorem 7] can be extended to those mixed strategies. Numerical examples that demonstrate the potential advantage of the generalized DF-CF [5, Theorem 7] with Gaussian signals over individual DF and CF schemes were provided in [26]. However, in that work, the SNR regions at which the generalized DF-CF reduces to either the DF or the $\mathrm{CF}$ scheme were not identified, nor those at which the generalization is guaranteed to yield strictly higher rates. In fact, the analysis of the generalized DF$\mathrm{CF}$ in [26] did prove the existence of distinct SNR operation regions and did not bound the maximum rate advantage.

In this paper, we consider a full-duplex single relay communication system in which the source transmissions are received by both the relay and the destination. The relay does not have its own message and its output depends either deterministically or stochastically on its observed signal [2], [3]. The destination uses a noisy combination of the source and relay signals to decode its intended message. We consider the generalized DF-CF scheme in [5, Theorem 7]. Since this scheme subsumes both DF and $\mathrm{CF}$, it will enable us to determine the appropriate relaying mode corresponding to a given SNR and will also enable us to obtain explicit expressions for SNR thresholds at which the relay ought to switch its operating mode, depending on the given channel realization. Numerical results show that, for quasi-static Rayleigh fading channel conditions, this selection can yield significant gains over fixed DF and CF. The SNR thresholds and the bound on the rate

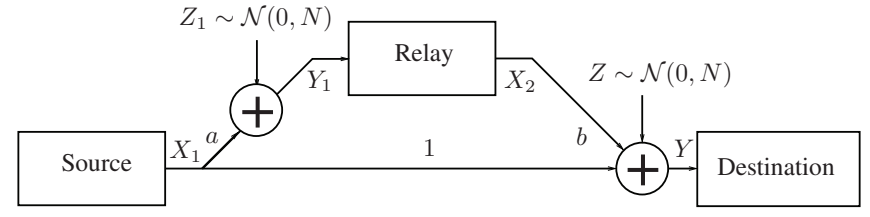

Fig. 1. Gaussian relay channel.

of the generalized scheme are obtained by particularizing the generalized DF-CF in [5, Theorem 7] to the case in which the source and relay signals are Gaussian and the relay channel is also Gaussian. The Karush-Kuhn-Tucker (KKT) optimality conditions corresponding to the problem of maximizing the achievable rate are then analyzed to draw insight into the generalized DF-CF scheme and to obtain the aforementioned results. A similar approach can be used to analyze the mixed DF-CF strategies in [25]-[27], but this is beyond the scope of this paper. To obtain a better understanding of the generalized DF-CF, we further analyze its maximum achievable rate and show that it is at most 0.5 bits per channel use (bpcu) higher than that achieved by the underlying DF and CF schemes.

The paper is organized as follows: Section II provides the system model. Section III provides explicit construction of the signals used by the generalized DF-CF scheme. The main results of the paper are derived in Section IV. Section V provides numerical examples and Section VI summarizes the paper. For convenience, most derivations are relegated to the appendices.

\section{SySTEM MODEL}

We consider the three-node Gaussian relay channel depicted in Figure 1. In this channel, the gain of the source-destination link is normalized, and the gains of the source-relay and the relay-destination links are denoted by the scalars $a$ and $b$, respectively. The source and relay transmit signals are denoted by $X_{1}$ and $X_{2}$, and the mutually independent additive Gaussian noises at the relay and destination are denoted by $Z_{1} \sim \mathcal{N}(0, N)$ and $Z \sim \mathcal{N}(0, N)$, respectively. The relay and destination received signals are respectively denoted by $Y_{1}$ and $Y$, and can be expressed as

$$
Y_{1}=a X_{1}+Z_{1}, \quad \text { and } \quad Y=X_{1}+b X_{2}+Z .
$$

The transmit power budgets at the source and relay are denoted by $P_{1}$ and $P_{2}$, respectively. Using this notation, we define $\gamma_{0} \triangleq \frac{P_{1}}{N}, \gamma_{1} \triangleq \frac{a^{2} P_{1}}{N}$, and $\gamma_{2} \triangleq \frac{b^{2} P_{2}}{N}$ to be the SNR of the source-destination, the source-relay, and the relay-destination links, respectively.

In the next section we will review the generalized DF-CF scheme in [5, Theorem 7] and apply it to the Gaussian channel in Figure 1 with Gaussian source and relay transmit signals.

\section{Generalization OF DF AND CF FOR GAUSSiAN RELAY CHANNELS}

\section{A. An information-theoretic background}

An achievable rate: In describing the codebook structure of the generalized DF-CF scheme, we will use the superscript $(\cdot)^{n}$ to denote the $n$-th extension of random variables. Source 
and relay length- $n$ codewords are constructed by superimposing respective DF-structured (partial) codewords on their CFstructured counterparts [5], [25]. The DF structure is generated in the standard way by using two random variables $U$ and $V$, where $U^{n}$ is the codeword representing one part of the message intended for the destination. Random partitions of $U^{n}$ are given by $V^{n}$, which are used by the relay to assist the decoding of $Y^{n}$ at the destination. The source codewords, $X_{1}^{n}$, are constructed by superimposing a length- $n$ vector representing the residual part of the message intended for the destination on $U^{n}$. The received signal at the relay is represented by $Y_{1}^{n}$ and a compressed description of it is given by $\hat{Y}_{1}^{n}$. The CF-structured codewords used to facilitate decoding are constructed by random partitioning of $\hat{Y}_{1}^{n}$. The length- $n$ vector representing the random partition is superimposed on $V^{n}$ to generate the relay transmitted codeword, $X_{2}^{n}$. Using this construction with sufficiently large $n$, the following rate is achieved [5]. The explicit construction will be given in Section III-B for the case of Gaussian codebooks.

$$
R_{G}^{*}=\sup \left\{\min \left\{R_{1}, R_{2}\right\}\right\},
$$

where the supremum is taken over joint distributions of $\left(U, V, \hat{Y}_{1}, X_{1}, X_{2}, Y_{1}, Y\right)$ satisfying

$$
I\left(\hat{Y}_{1} ; Y_{1} \mid Y, X_{2}, U\right) \leq I\left(X_{2} ; Y \mid V\right),
$$

and the rates $R_{1}$ and $R_{2}$ are given by

$$
\begin{aligned}
& R_{1}=I\left(X_{1} ; Y, \hat{Y}_{1} \mid X_{2}, U\right)+I\left(U ; Y_{1} \mid X_{2}, V\right), \\
& R_{2}=I\left(X_{1}, X_{2} ; Y\right)-I\left(\hat{Y}_{1} ; Y_{1} \mid X_{1}, U, X_{2}, Y\right) .
\end{aligned}
$$

The cut-set bound: An upper bound on the capacity, $C$, of a general multi-terminal channel is given by the cut-set bound in [3]. This bound is attained by most relaying strategies that are known to achieve capacity for specific channel models [5], [7], [19]-[22]. Instances in which this bound was used to compute the capacity of various relay networks are provided in [18], [28]. Applying this bound to the standard three-node relay channel yields [5, Theorem 4]

$$
C \leq \sup _{p\left(x_{1}, x_{2}\right)} \min \left\{I\left(X_{1} ; Y, Y_{1} \mid X_{2}\right), I\left(X_{1}, X_{2} ; Y\right)\right\} .
$$

\section{B. Application to Gaussian signals}

We now derive the expressions corresponding to (2) and (3) when the relay channel and the signals are Gaussian. To map the codebook structure described in [5], [25] to Gaussian signals, we define the power partitions $\left\{\alpha_{i}\right\}_{i=0}^{2}$ to be used at the source to construct $U$, a scaled version of $V$ and $X_{1}$, and define $\left\{\beta_{j}\right\}_{j=0}^{1}$ to be used at the relay to construct another scaled version of $V$ and $X_{2}$, respectively. The power partitions satisfy $\alpha_{i}, \beta_{j} \geq 0, i=0,1,2, j=0,1, \sum_{i=0}^{2} \alpha_{i}=1$, and $\sum_{j=0}^{1} \beta_{j}=1$. To proceed with the description of Gaussian signals, we let $V \sim \mathcal{N}(0,1), V_{1}=\sqrt{\alpha_{0} P_{1}} V$, and $V_{2}=\sqrt{\beta_{0} P_{2}} V$.

The construction in [5] implies that each signal $U$ can be expressed as the superposition of a (base) component $V_{1}$ and another independent (incremental) component, $X_{11}$. The signals $X_{1}$ and $X_{2}$ are constructed similarly using base components $U$ and $V_{2}$ and incremental components $X_{12}$ and
$X_{22}$, respectively. Using the approach in [7] and [6], the auxiliary signal $\hat{Y}_{1}$ can be expressed as the superposition of $Y_{1}, X_{2}$ and $U$, as a base component, and a statistically independent estimation noise $Z^{\prime}$, as an incremental component. In particular, we can write

$$
\begin{aligned}
U & =V_{1}+X_{11}, & & X_{1}=U+X_{12}, \\
X_{2} & =V_{2}+X_{22}, & & \hat{Y}_{1}=Y_{1}+X_{2}+U+Z^{\prime},
\end{aligned}
$$

where $Z^{\prime} \sim \mathcal{N}\left(0, N^{\prime}\right)$. Note that, in this construction, $N^{\prime}$ is a system parameter, $V, X_{11}$ and $X_{22}$ are mutually statistically independent, and $U$ and $X_{12}$ are statistically independent; $X_{1}$ and $X_{2}$ are correlated through $V$. The source uses the power fraction $\alpha_{0} P_{1}$ to transmit $V_{1}$, the power fraction $\alpha_{1} P_{1}$ to transmit $X_{11}$ and the power fraction $\alpha_{2} P_{1}$ to transmit $X_{12}$. The relay uses the power fraction $\beta_{0} P_{2}$ to transmit $V_{2}$ and the power fraction $\beta_{1} P_{2}$ to transmit $X_{22}$.

\section{The Generalized DF-CF: Achievable Rate And ANALYSIS}

In this section, we will use the construction for the signals in Section III-B to analyze the maximum rate that the generalized DF-CF scheme can achieve when the channel in Figure 1 and the signals in (5) are Gaussian distributed. Our first step is to particularize the rate given in (2) to obtain the corresponding expressions for the Gaussian case. We then use these expressions to formulate the problem of maximizing the achievable rate of the generalized DF-CF scheme as an optimization problem. In the remainder of the section, we will draw insight into the structure of this problem by studying its KKT conditions in various SNR regions.

Particularizing the rate expressions in (2) to the Gaussian case yields the following result.

Proposition 1: Applying the generalized DF-CF scheme with Gaussian signal components to the Gaussian relay channel in Figure 1 and using $\mathcal{C}(x) \triangleq \frac{1}{2} \log _{2}(1+x)$, and $\gamma^{\prime} \triangleq N^{\prime} / N$ yields:

$$
\begin{aligned}
R_{G}^{*}= & \max _{\left\{\alpha_{i}\right\}_{i=0}^{2},\left\{\beta_{j}\right\}_{j=0}^{1}, \gamma^{\prime}} \min \left\{R_{1}, R_{2}\right\} \\
& \text { subject to } \\
& \gamma^{\prime} \geq \frac{\left(1+\alpha_{2}\left(\gamma_{0}+\gamma_{1}\right)\right)\left(1+\left(\alpha_{1}+\alpha_{2}\right) \gamma_{0}\right)}{\left(1+\alpha_{2} \gamma_{0}\right) \beta_{1} \gamma_{2}}, \\
& R_{1}=\mathcal{C}\left(\frac{\alpha_{2} \gamma_{1}}{1+\gamma^{\prime}}+\alpha_{2} \gamma_{0}\right)+\mathcal{C}\left(\frac{\alpha_{1} \gamma_{1}}{1+\alpha_{2} \gamma_{1}}\right), \\
& R_{2}=\mathcal{C}\left(\gamma_{0}+\gamma_{2}+2 \sqrt{\alpha_{0} \beta_{0} \gamma_{0} \gamma_{2}}\right)-\mathcal{C}\left(\gamma^{\prime-1}\right), \\
& \sum_{i=0}^{2} \alpha_{i}=1, \sum_{j=0}^{1} \beta_{j}=1 \\
& \alpha_{i} \geq 0, \beta_{j} \geq 0, \forall i, j .
\end{aligned}
$$

Proof: The derivation resembles the one in [26, Section III-D], and is omitted for brevity.

In subsequent analysis, we will seek solutions of the optimization problem in (6) for different SNR regions. We begin by recasting (6) in a more convenient form. First, we introduce a new variable $t \geq 1$ such that $\frac{1}{2} \log (t)$ is a lower bound on $R_{1}$ and $R_{2}$. Then, invoking the fact that the $\log (\cdot)$ function is monotonically increasing, the optimization in (6) is recast as 
$\max \quad t$,

subject to $\quad g_{i}\left(t, \alpha_{0}, \alpha_{2}, \beta_{0}, \gamma^{\prime}\right) \leq 0, \quad i=1, \ldots, 8$

where, using $\sum_{i=0}^{2} \alpha_{i}=1$ and $\sum_{j=0}^{1} \beta_{j}=1$ to eliminate $\alpha_{1}$ and $\beta_{1},\left\{g_{i}\right\}_{i=1}^{8}$ can be defined as

$$
\begin{aligned}
& g_{1}\left(t, \alpha_{0}, \alpha_{2}, \beta_{0}, \gamma^{\prime}\right) \triangleq t-\left(1+\frac{\alpha_{2} \gamma_{1}}{1+\gamma^{\prime}}+\alpha_{2} \gamma_{0}\right) \frac{1+\left(1-\alpha_{0}\right) \gamma_{1}}{1+\alpha_{2} \gamma_{1}} \\
& g_{2}\left(t, \alpha_{0}, \alpha_{2}, \beta_{0}, \gamma^{\prime}\right) \triangleq t-\left(1+\gamma_{0}+\gamma_{2}+2 \sqrt{\alpha_{0} \beta_{0} \gamma_{0} \gamma_{2}}\right) \frac{\gamma^{\prime}}{1+\gamma^{\prime}} \\
& g_{3}\left(t, \alpha_{0}, \alpha_{2}, \beta_{0}, \gamma^{\prime}\right) \triangleq 1+\frac{\alpha_{2} \gamma_{1}}{1+\alpha_{2} \gamma_{0}}-\frac{\left(1-\beta_{0}\right) \gamma_{2}}{1+\left(1-\alpha_{0}\right) \gamma_{0}} \gamma^{\prime} \\
& g_{4}\left(t, \alpha_{0}, \alpha_{2}, \beta_{0}, \gamma^{\prime}\right) \triangleq-\alpha_{2} \\
& g_{5}\left(t, \alpha_{0}, \alpha_{2}, \beta_{0}, \gamma^{\prime}\right) \triangleq-\alpha_{0} \\
& g_{6}\left(t, \alpha_{0}, \alpha_{2}, \beta_{0}, \gamma^{\prime}\right) \triangleq \alpha_{0}+\alpha_{2}-1 \\
& g_{7}\left(t, \alpha_{0}, \alpha_{2}, \beta_{0}, \gamma^{\prime}\right) \triangleq \beta_{0}-1 \\
& g_{8}\left(t, \alpha_{0}, \alpha_{2}, \beta_{0}, \gamma^{\prime}\right) \triangleq-\beta_{0}
\end{aligned}
$$

The optimization in (7) is not convex. Hence, the KKT conditions are not sufficient for optimality. However, if a point is a local minimum of (7) and if that point is regular (i.e., the gradients of the active constraints are linearly independent), then the KKT conditions are necessary for optimality [29, Proposition 3.3.1]. In our analysis we will consider the KKT system of (7); the regularity of the solutions that we obtain can be verified by inspection.

Using $\lambda_{i}$ to denote the Lagrange multiplier corresponding to the $i$-th constraint in $(7 \mathrm{~b}), i=1, \ldots, 8$, the KKT system can be written as

$$
\begin{aligned}
& \lambda_{1}+\lambda_{2}-1=0 \text {, } \\
& \frac{\lambda_{1} \gamma_{1}}{1+\alpha_{2} \gamma_{1}}\left(1+\alpha_{2} \gamma_{0}+\frac{\alpha_{2} \gamma_{1}}{1+\gamma^{\prime}}\right)-\frac{\lambda_{2} \gamma^{\prime}}{1+\gamma^{\prime}} \sqrt{\frac{\gamma_{0} \gamma_{2} \beta_{0}}{\alpha_{0}}} \\
& -\frac{\lambda_{3} \gamma_{0} \gamma_{2} \gamma^{\prime}\left(1-\beta_{0}\right)}{\left(1+\left(1-\alpha_{0}\right) \gamma_{0}\right)^{2}}-\lambda_{5}+\lambda_{6}=0, \\
& \frac{\lambda_{1}\left(1+\gamma_{1}\left(1-\alpha_{0}\right)\right)\left(\gamma_{0}\left(1+\gamma^{\prime}\right)-\gamma_{1} \gamma^{\prime}\right)}{\left(1+\gamma^{\prime}\right)\left(1+\alpha_{2} \gamma_{1}\right)^{2}} \\
& -\frac{\lambda_{3} \gamma_{1}}{\left(1+\alpha_{2} \gamma_{0}\right)^{2}}+\lambda_{4}-\lambda_{6}=0 \text {, } \\
& \frac{\lambda_{2} \gamma^{\prime}}{1+\gamma^{\prime}} \sqrt{\frac{\gamma_{0} \gamma_{2} \alpha_{0}}{\beta_{0}}}-\frac{\lambda_{3} \gamma_{2} \gamma^{\prime}}{1+\left(1-\alpha_{0}\right) \gamma_{0}}-\lambda_{7}+\lambda_{8}=0 \text {, } \\
& \frac{\lambda_{1} \gamma_{1} \alpha_{2}\left(1+\gamma_{1}\left(1-\alpha_{0}\right)\right)}{\left(1+\gamma^{\prime}\right)^{2}\left(1+\gamma_{1} \alpha_{2}\right)}-\frac{\lambda_{2}\left(1+\gamma_{0}+\gamma_{2}+2 \sqrt{\alpha_{0} \beta_{0} \gamma_{0} \gamma_{2}}\right)}{\left(1+\gamma^{\prime}\right)^{2}} \\
& -\frac{\lambda_{3} \gamma_{2}\left(1-\beta_{0}\right)}{1+\gamma_{0}\left(1-\alpha_{0}\right)}=0 \\
& g_{i}\left(t, \alpha_{0}, \alpha_{2}, \beta_{0}, \gamma^{\prime}\right) \leq 0, \quad \lambda_{i} g_{i}\left(t, \alpha_{0}, \alpha_{2}, \beta_{0}, \gamma^{\prime}\right)=0 \text {, } \\
& \lambda_{i} \geq 0, \quad i=1,2, \ldots, 8 \text {. }
\end{aligned}
$$

To study the KKT system, we will determine the values of $\alpha_{0}, \alpha_{2}, \beta_{0}$ and $\gamma^{\prime}$ for different SNR regions. We begin by providing achievable rates of the DF and CF schemes for the Gaussian channel in Figure 1. The derivation of these rates is omitted for brevity. For the DF scheme, the maximum achievable rate is achieved with Gaussian signals [6, Proposition 2] and is given by

$$
R_{D F}^{*}=\max _{\left\{\rho_{i}\right\}_{i=0}^{1}} \min \left\{R_{D F, 1}, R_{D F, 2}\right\},
$$

where $R_{D F, 1}=\mathcal{C}\left(\rho_{1} \gamma_{1}\right)$ and $R_{D F, 2}=\mathcal{C}\left(\gamma_{0}+\gamma_{2}+\right.$ $\left.2 \sqrt{\rho_{0} \gamma_{0} \gamma_{2}}\right), \rho_{0}$ and $\rho_{1}$ satisfy $\sum_{i=0}^{1} \rho_{i}=1$, and $\sqrt{\rho_{0}}=$ $\mathrm{E}\left\{X_{1} X_{2}\right\}$, where $\mathrm{E}\{\cdot\}$ denotes the expectation operator; cf. [5, Theorem 1]. In contrast with the DF scheme, in the CF scheme it is not known if using Gaussian signalling achieves its maximum rate. However, the maximum rate achieved with Gaussian signals is given by

$$
R_{C F}^{*}=\mathcal{C}\left(\frac{\gamma_{0}+\gamma_{1} \gamma_{2}}{\left(1+\gamma_{0}+\gamma_{1}+\gamma_{2}\right)}\right) \text {, }
$$

where the normalized variance of the estimation noise that enables this rate to be achieved is

$$
\gamma_{C F}^{\prime *} \triangleq \frac{\left(1+\gamma_{0}+\gamma_{1}\right)}{\gamma_{2}} .
$$

Remark 1: Let $\gamma_{i} \in(0, \infty), i=0,1,2$. Then, setting $\alpha_{2}=0$ and $\beta_{0}=1$, the generalized DF-CF scheme yields the maximum rate of the DF scheme, $R_{D F}^{*}$. Furthermore, setting $\alpha_{2}=1$ and $\beta_{1}=1$, the generalized DF-CF scheme yields the maximum rate of the $\mathrm{CF}$ scheme with Gaussian signals, $R_{C F}^{*}$. In particular, $\left.R_{G}^{*}\right|_{\substack{\alpha_{2}=0 \\ \beta_{1}=0}}=R_{D F}^{*}$, and $\left.R_{G}^{*}\right|_{\substack{\alpha_{2}=1 \\ \beta_{1}=1}}=R_{C F}^{*}$, with $\left.\gamma^{* *}\right|_{\substack{\alpha_{2}=1 \\ \beta_{1}=1}}=\gamma_{C F}^{*}$.

Proof: Direct substitution in (6) yields the statements of the remark.

We now analyze the rates yielded by the problem in (6) for different choices of $\gamma_{0}, \gamma_{1}$ and $\gamma_{2}$. We will consider the case of $\gamma_{0}\left(1+\gamma_{2}\right)<\gamma_{1}<\infty$ in Section IV-A, the case of $\gamma_{1} \leq \gamma_{0}$ in Section IV-B. The remaining case in which $\gamma_{1} \in$ $\left(\gamma_{0}, \gamma_{0}\left(1+\gamma_{2}\right)\right]$ has proved difficult to analyze. To circumvent this difficulty, in Section IV-C we will consider SNRs that yield $R_{D F}^{*}=R_{C F}^{*}$. Those SNRs can be readily verified to belong to the region $\left(\gamma_{0}, \gamma_{0}\left(1+\gamma_{2}\right)\right]$. In Section IV-C we will touch upon two asymptotic cases, viz., $\gamma_{2} \rightarrow \infty$ and $\gamma_{0} \rightarrow 0$. In Section IV-D we will provide an upper bound on the gain of the generalized DF-CF scheme.

\section{A. The case of $\gamma_{0}\left(1+\gamma_{2}\right)<\gamma_{1}<\infty$}

In this region $a>\sqrt{1+\frac{b^{2} P_{1}}{N_{1}}}$. For this case, we have the following result.

Theorem 1: When the channel gains in Figure 1 satisfy $\gamma_{1}>\gamma_{0}\left(1+\gamma_{2}\right)$, the generalized DF-CF scheme using Gaussian signals reduces to the DF scheme. In particular, $R_{G}^{*}=R_{D F}^{*}$.

Proof: See Appendix A.

Hence, if the channel gain of the source-relay link is sufficiently greater than the gain of source-destination link, the generalized DF-CF with Gaussian signals does not yield rates higher than those achieved by the DF scheme.

Remark 2: By comparing (17) with (18) in Appendix A, it can be seen that when $\gamma_{0}\left(1+\gamma_{2}\right)<\gamma_{1}<\gamma_{0}+\gamma_{2}$, $R_{1}<R_{2}$; i.e., $R_{1}$ is the constraining rate. In this case, $\beta_{0}$ can be set to be less than 1 without reducing the achievable rate of the generalized DF-CF scheme. Setting $\beta_{0}<1$ 
implies that the relay uses a fraction $\beta_{1}>0$ of its power to transmit its estimation of the received signal. Setting $\alpha_{1}=1$ and restricting $R_{2}$ to be greater than or equal to $R_{1}$ yields $\beta_{1} \leq \frac{1+\gamma_{0}}{1+\gamma_{1}}\left(1-\frac{\gamma_{1}-\gamma_{0}}{\gamma_{2}}\right)$.

This remark implies that when $\gamma_{0}\left(1+\gamma_{2}\right)<\gamma_{1}<\gamma_{0}+\gamma_{2}$, the relay does not need to use all its power for the maximum generalized DF-CF rate to be achieved. We suspect that the additional power might be used to yield a rate advantage for networks with multiple destinations.

Having considered the case of $\gamma_{0}\left(1+\gamma_{2}\right)<\gamma_{1}$, next we will consider the case of $\gamma_{1} \leq \gamma_{0}$.

\section{B. The case of $\gamma_{1} \leq \gamma_{0}$}

When $\gamma_{1} \leq \gamma_{0}, a \leq 1$ and the result for this case is given in the following theorem.

Theorem 2: When the gains in Figure 1 satisfy $\gamma_{1} \leq \gamma_{0}$, the generalized DF-CF scheme using Gaussian signals reduces to the corresponding $\mathrm{CF}$ scheme. In particular, $R_{G}^{*}=R_{C F}^{*}$.

Proof: See Appendix B.

Hence, when the signals are Gaussian and $a \leq 1$, the rate yielded by the generalized DF-CF scheme is not higher than the rate of the corresponding $\mathrm{CF}$ scheme.

So far we have considered the cases of $\gamma_{1}>\gamma_{0}\left(1+\gamma_{2}\right)$ and $\gamma_{1} \leq \gamma_{0}$. Unfortunately, the case of $\gamma_{0}<\gamma_{1} \leq \gamma_{0}\left(1+\gamma_{2}\right)$ is rather difficult to analyze. To gain insight into this case, we consider a subset of SNRs that falls thereunder. In particular, in the next section we will focus on the case of $\gamma_{1}\left(1+\gamma_{1}\right)=$ $\gamma_{0}\left(1+\gamma_{0}+\gamma_{2}\right)$, which will be shown to correspond to the case of $R_{D F}^{*}=R_{C F}^{*}$.

\section{The case of SNRs yielding $R_{D F}^{*}=R_{C F}^{*}$}

Here we will show that when $R_{D F}^{*}=R_{C F}^{*}$, the rate yielded by the generalized DF-CF scheme is strictly higher than the rate yielded by either scheme. We begin by stating the following lemma.

Lemma 1: For the Gaussian channel shown in Figure 1, the DF scheme and the CF scheme using Gaussian signals yield the same maximum achievable rate if and only if

$$
\gamma_{1}\left(1+\gamma_{1}\right)=\gamma_{0}\left(1+\gamma_{0}+\gamma_{2}\right)
$$

Proof: See Appendix C-A.

An important implication of this lemma is given in the following corollary.

Corollary 1: When $\gamma_{1}\left(1+\gamma_{1}\right)>\gamma_{0}\left(1+\gamma_{0}+\gamma_{2}\right), R_{D F}^{*}>$ $R_{C F}^{*}$ and when $\gamma_{1}\left(1+\gamma_{1}\right)<\gamma_{0}\left(1+\gamma_{0}+\gamma_{2}\right), R_{D F}^{*}<R_{C F}^{*}$.

Proof: See Appendix C-B.

Corollary 1 provides an SNR threshold below which DF is more advantageous than $\mathrm{CF}$, and above which $\mathrm{CF}$ is more advantageous than DF. This result will be used to develop a DF/CF switching scheme which will be shown to yield significant rate advantage over individual schemes under quasi-static Rayleigh fading channel conditions.

Using Lemma 1, we have the following result.

Theorem 3: Suppose that the SNRs of the Gaussian channel in Figure 1 are finite and bounded away from zero, i.e., $0<$ $\gamma_{0}, \gamma_{1}, \gamma_{2}<\infty$. If $\gamma_{i}, i=0,1,2$, satisfy (12), then there exist power partitions, $\left\{\alpha_{i}\right\}_{i=0}^{2}$ and $\left\{\beta_{j}\right\}_{j=0}^{1}$, and normalized estimation noise, $\gamma^{\prime}$, such that the generalized DF-CF using Gaussian signals provides a higher rate than the corresponding DF and CF schemes.

Proof: See Appendix D.

Theorem 3 shows that for SNRs satisfying the condition of Lemma 1, the generalized DF-CF using Gaussian signals yields strictly higher rates than the corresponding DF and $\mathrm{CF}$ schemes.

So far we have restricted our attention to strictly positive finite SNRs. In the following remarks we will consider two extreme cases, namely, $\gamma_{2} \rightarrow \infty$ and $\gamma_{0} \rightarrow 0$.

Remark 3: When $\gamma_{2} \rightarrow \infty$ and $0<\gamma_{0}, \gamma_{1}<\infty$, the generalized DF-CF scheme using Gaussian signals reduces to the corresponding $\mathrm{CF}$ scheme and achieves the capacity of the relay channel; i.e., $R_{G}^{*}=R_{C F}^{*}=C$.

The proof of this remark uses the fact that the Gaussian distribution maximize the cut-set bound [6] for the considered Gaussian channel and that this bound is achieved by the $\mathrm{CF}$ scheme using Gaussian signals when $\gamma_{2} \rightarrow \infty$. A similar observation has been made independently in [7]. The fact that $\mathrm{CF}$ is a special case of the generalized CF-DF yields the result.

Remark 4: When $\gamma_{0} \rightarrow 0,0<\gamma_{1}$ and $\gamma_{2}<\infty$, the generalized DF-CF scheme reduces to DF, and achieves the capacity of the channel in Figure 1. In particular, $R_{G}^{*}=R_{D F}^{*}=C$. $\square$

To prove this remark, we invoke the fact that the cutset bound is maximized by the Gaussian distribution and that Gaussian signals maximize the rate achieved by the DF scheme for the considered relay channel. Direct substitution for $\gamma_{0} \rightarrow 0$ in the corresponding rate expressions show that the DF scheme meets the cut-set bound and hence achieves capacity. The fact that DF is a special case of the generalized CF-DF scheme yields the result.

Having analyzed the maximum rate that can be achieved by the generalized DF-CF scheme in various SNR regions, we next provide an upper bound on the gap between the maximum achievable rate of the generalized scheme and that of individual DF and $\mathrm{CF}$ schemes.

\section{An upper bound on the gain of the generalized DF-CF}

An upper bound on the gain of the generalized DF-CF using Gaussian signals over the corresponding DF and CF schemes is provided in the following theorem.

Theorem 4: Using Gaussian signals, the maximum rate achieved by the generalized DF-CF scheme is at most $0.5 \mathrm{bpcu}$ higher than that achieved by individual DF and CF schemes.

Proof: See Appendix E.

This theorem implies that, despite the inherent coding and decoding complexity of the generalized DF-CF, its rate advantage over DF and CF is relatively small. This indicates that the practical benefit of the analysis of this scheme is to enable appropriate selection of either DF or CF relaying depending on the given channel realization.

In the next section, we will provide numerical confirmation for the analytical findings obtained in this section. We will also provide an example in which this analysis is exploited to adapt the relay operation mode to the channel gains. 


\section{NumericAl RESUltS}

For numerical analysis, we note that the maximum DF rate, $R_{D F}^{*}$, can be obtained from (9), by setting $\rho_{0}^{*}=0$, or such that $R_{D F, 1}=R_{D F, 2}$, depending on the values of $\gamma_{i}, i=0,1,2$. The maximum CF rate, $R_{C F}^{*}$, is evaluated using (10), and the maximum generalized DF-CF rate, $R_{G}^{*}$, is evaluated by using the KKT conditions corresponding to (6) to reduce the search for $\gamma^{\prime *}$ and the optimal power partitions to three parameters, viz., $\alpha_{0}, \alpha_{2}, \beta_{0} \in[0,1]$. Using the KKT system (8), it can be shown that, when $\left\{\alpha_{i}\right\}_{i=0}^{2}>0$ and $\left\{\beta_{j}\right\}_{j=0}^{1}>0, R_{1}=R_{2}$ and (6b) holds with equality. Using these results, the search for the optimal parameters can be simplified by expressing $\alpha_{2}$ as a function of $\gamma^{\prime}, \alpha_{0}$ and $\beta_{0}$. The cases in which a subset of $\left\{\alpha_{i}\right\}_{i=0}^{2}$ and $\left\{\beta_{j}\right\}_{j=0}^{1}$ are zero can be readily inferred by the continuity of the rate expressions in the power partitions. The cut-set bound is calculated using the expression in Theorem 4 in [5].

In Figure 2, the SNR of the source-destination link is set to be $\gamma_{0}=5 \mathrm{~dB}$ and that of the relay-destination link is set to be $\gamma_{2}=5.5 \mathrm{~dB}$, which yields $\gamma_{0}\left(1+\gamma_{2}\right)=11.5783 \mathrm{~dB}$.

Figure 2 shows that, in agreement with Theorem 1, for $\gamma_{1}>11.5783 \mathrm{~dB}$, the maximum rate of the generalized DF$\mathrm{CF}$ scheme, $R_{G}^{*}$, coincides with the maximum rate of the $\mathrm{DF}$ scheme, $R_{D F}^{*}$. Similarly, in agreement with Theorem 2, for $\gamma_{1} \leq 5 \mathrm{~dB}, R_{G}^{*}$ coincides with the maximum rate of the $\mathrm{CF}$ scheme, $R_{C F}^{*}$. We note that Theorem 1 and 2 provide SNR conditions that are only sufficient for the generalized DF-CF scheme to reduce to DF or CF, respectively. However, these conditions are not necessary. In fact, we observe that the generalized DF-CF scheme reduces to DF and CF in the neighbourhood of the inner boundary of $\gamma_{0}<\gamma_{1} \leq \gamma_{0}\left(1+\gamma_{2}\right)$.

To investigate the performance of the generalized DF-CF scheme when $\gamma_{0}<\gamma_{1} \leq \gamma_{0}\left(1+\gamma_{2}\right)$, in Figure 2, we plot a magnified version of the region spanned by $\gamma_{1} \in[6.3,6.6]$. As asserted by Theorem 3, it can be seen that, when $\gamma_{1}=$ $6.496377 \mathrm{~dB}, \gamma_{1}\left(1+\gamma_{1}\right)=\gamma_{0}\left(1+\gamma_{0}+\gamma_{2}\right)$, resulting in $R_{1}=$ $R_{2}$. This setting yields $R_{D F}^{*}=R_{C F}^{*}=1.22486$ bpcu. At this SNR, the generalized DF-CF scheme yields a rate advantage $\Delta_{G}=R_{G}^{*}-R_{D F}^{*}=2.62 \times 10^{-3}$ bpcu. This rate advantage is obtained by setting $\alpha_{0}=0.004, \alpha_{1}=0.762621, \alpha_{2}=$ $1-\alpha_{1}-\alpha_{0}, \beta_{0}=0.179$, and $\beta_{1}=1-\beta_{0}$. In agreement with Theorem 4 , the gain offered by the generalized DF-CF scheme is strictly less than $0.5 \mathrm{bpcu}$. Similar to the numerical results reported for the generalization in [27], the rate advantage of the generalization considered herein is marginal. The cut-set bound at these particular SNRs is $1.54503 \mathrm{bpcu}$ and the gap to the generalized DF-CF rate is 0.31755 bpcu. However, this does not preclude the possibility that the generalized DF-CF might yield higher rate gains for more general networks; cf. Remark 2.

In Figure 3 we verify Remarks 3 and 4. In Figure 3(a), we set $\gamma_{0}=1 \mathrm{~dB}$ and $\gamma_{1}=5 \mathrm{~dB}$. In accordance with Remark 3, it can be seen that as $\gamma_{2}$ becomes sufficiently large, $R_{G}^{*}$ coincides with $R_{C F}^{*}$ and with the cut-set bound. Figure 3(b) is obtained by setting $\gamma_{1}=10 \mathrm{~dB}$ and $\gamma_{2}=5 \mathrm{~dB}$. As stated in Remark 4, for small $\gamma_{0}, R_{G}^{*}$ coincides with $R_{D F}^{*}$ and with the cut-set bound.

Finally, we provide a numerical example to expose the

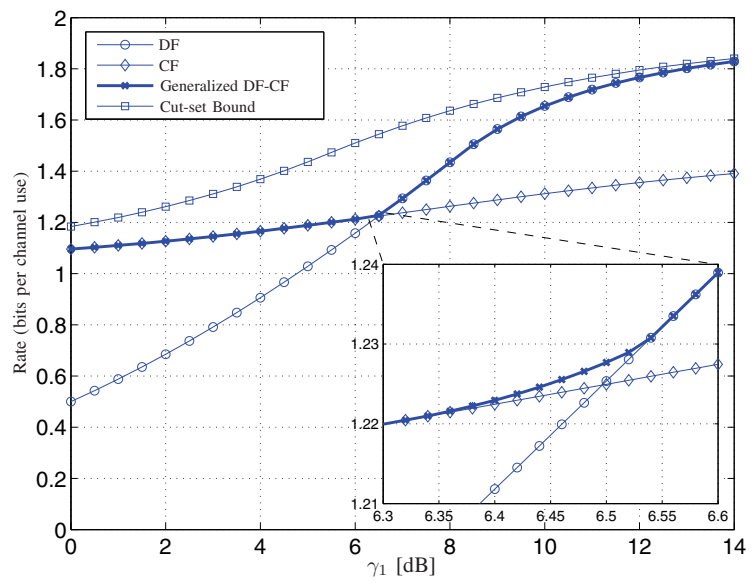

Fig. 2. Maximum achievable rate of the generalized DF-CF scheme.

practical implications of the analysis of the generalized DF$\mathrm{CF}$ scheme. We consider a quasi-static frequency-flat Rayleigh fading relay channel in which the channel coefficients take on random values, but are held constant for the entire signalling duration. The coefficients are drawn from the standard zero mean Gaussian distribution with the variance adjusted to yield two instances of average SNRs, $\bar{\gamma}_{i}=\mathrm{E}\left\{\gamma_{i}\right\}, i=0,1,2$. In both instances $\bar{\gamma}_{0}=5 \mathrm{~dB}$ and $\bar{\gamma}_{1}$ ranges between 0 and $20 \mathrm{~dB}$. In the first instance $\bar{\gamma}_{2}=5.5 \mathrm{~dB}$, whereas in the second instance $\bar{\gamma}_{2}=10 \mathrm{~dB}$. For both instances, we assume that the instantaneous channel gains, and hence $\left\{\gamma_{i}\right\}_{i=0}^{2}$, are available at the source, relay and destination, and we compare the following three schemes: the standard DF and CF schemes and a DF/CF switching scheme. In the standard schemes the relay uses either DF or CF relaying regardless of the channel realization, whereas in the switching scheme, the relay uses Corollary 1 to select its operation mode depending on the current realization of $\left\{\gamma_{i}\right\}_{i=0}^{2}$. From Figure 4(a) and 4(b), it can be seen that enabling the relay to switch between DF and $\mathrm{CF}$ yields a significant gain over either scheme. For the first instance, Figure 4(a) shows a gain of $4 \mathrm{~dB}$, when the DF/CF switching scheme is used and the rate is $1.1 \mathrm{bpcu}$. The second instance is considered in Figure 4(b) and the gain of the DF/CF switching scheme is $3 \mathrm{~dB}$, when the rate is $1.3 \mathrm{bpcu}$.

\section{CONCLUSION}

In this paper, we investigated the generalized DF-CF scheme developed in Theorem 7 in [5] when Gaussian signals are used for signalling over scalar power-constrained Gaussian memoryless channels. Gaussian signals are generally not known to be optimal for either the CF or the generalized scheme and higher rates might be achievable if the input signals were optimally distributed. When the signals are restricted to be Gaussian, the generalized DF-CF scheme:

- reduces to CF, and outperforms DF, if either the SNR of the source-relay link is less than or equal to the SNR of the source-destination link, or if the relay transmit power is sufficiently high. In the latter case the CF scheme achieves capacity; 


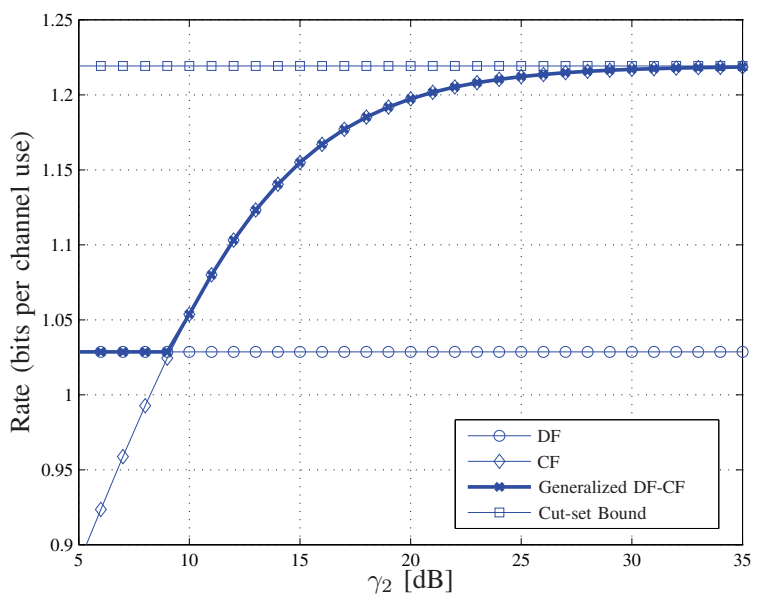

(a) Generalized DF-CF reduces to CF and achieves capacity

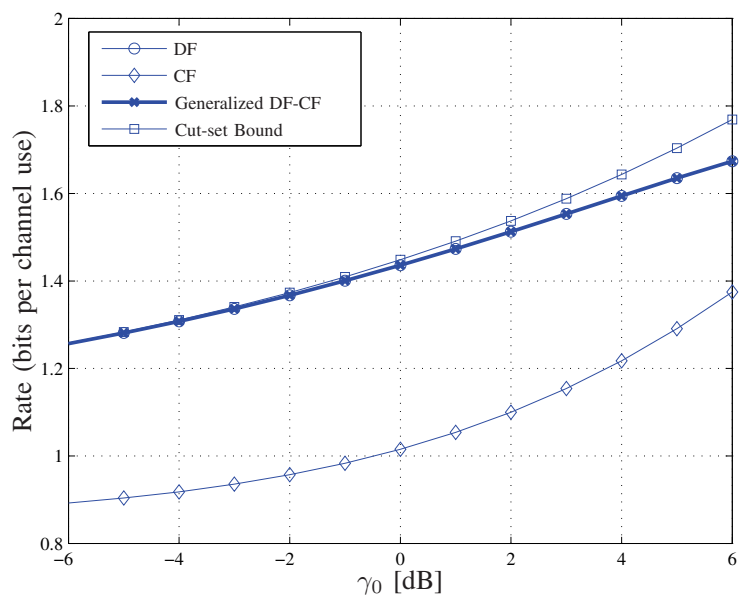

(b) Generalized DF-CF reduces to DF and achieves capacity

Fig. 3. Asymptotic optimality of the generalized DF-CF scheme.

- reduces to DF, and outperforms CF, when the SNR of the source-relay link is sufficiently higher than that of the source-destination link, or when the SNR of the sourcedestination link is sufficiently low. In the latter case the DF scheme achieves capacity; and

- achieves a strictly higher rate than the corresponding DF and $\mathrm{CF}$ schemes, when these schemes yield the same rate.

In addition, we have shown that the advantage of the generalized DF-CF scheme over individual DF and CF schemes is upper bounded by $0.5 \mathrm{bpcu}$. Finally, we exploited the analysis of the generalized DF-CF to provide a DF/CF switching scheme that was shown to yield substantial rate gains over standard DF and CF schemes under quasi-static Rayleigh fading channel conditions.

\section{APPENDIX A PROOF OF THEOREM 1}

The proof of this theorem relies on the following result. Lemma 2: When $\gamma_{1}>\gamma_{0}\left(1+\gamma_{2}\right), \gamma^{\prime}$ must satisfy

$$
\gamma^{\prime}>\frac{\gamma_{0}}{\gamma_{1}-\gamma_{0}} \text {. }
$$

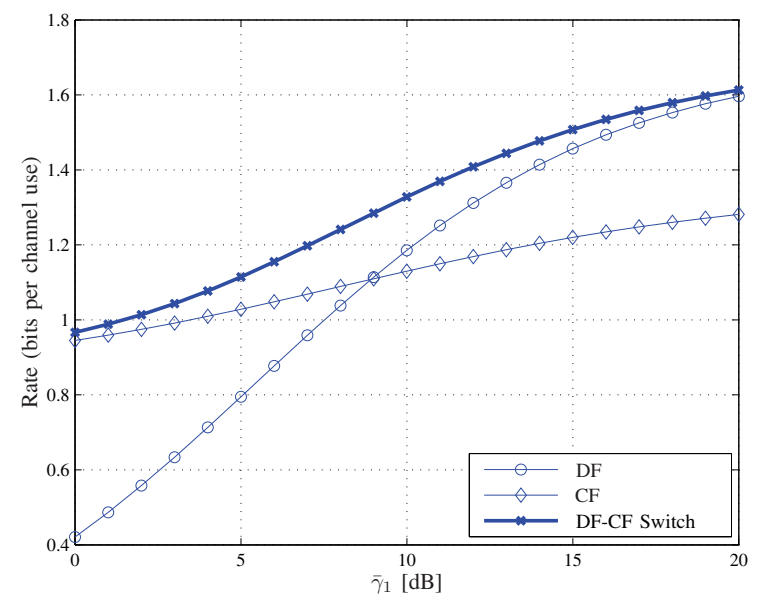

(a) $\bar{\gamma}_{0}=5 \mathrm{~dB}, \bar{\gamma}_{2}=5.5 \mathrm{~dB}$

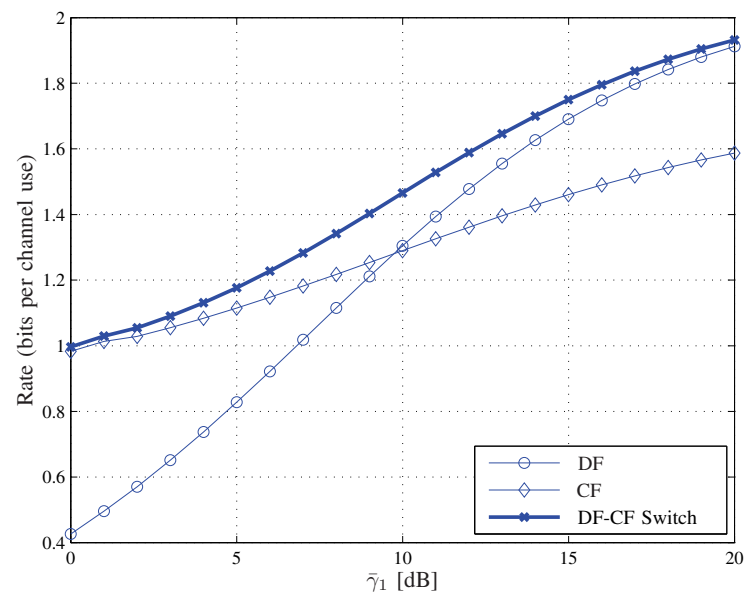

(b) $\bar{\gamma}_{0}=5 \mathrm{~dB}, \overline{\gamma_{2}}=10 \mathrm{~dB}$

Fig. 4. Maximum achievable rate in state-static Rayleigh fading channel.

Proof: We proceed by contradiction. Suppose that $\gamma^{\prime} \leq \frac{\gamma_{0}}{\gamma_{1}-\gamma_{0}}$. In this case, the constraint in (6b) yields $\frac{\left(1+\alpha_{2}\left(\gamma_{0}+\gamma_{1}\right)\right)\left(1+\left(\alpha_{1}+\alpha_{2}\right) \gamma_{0}\right)}{\left(1+\alpha_{2} \gamma_{0}\right) \beta_{1} \gamma_{2}} \leq \frac{\gamma_{0}}{\gamma_{1}-\gamma_{0}}$, which, by the fact that $\beta_{1} \leq 1$, implies that

$$
\begin{aligned}
\left(1+\alpha_{2}\left(\gamma_{0}+\gamma_{1}\right)\right)\left(1+\left(\alpha_{1}+\right.\right. & \left.\left.\alpha_{2}\right) \gamma_{0}\right) \\
& \leq \frac{\gamma_{0}}{\gamma_{1}-\gamma_{0}}\left(1+\alpha_{2} \gamma_{0}\right) \gamma_{2}
\end{aligned}
$$

Manipulating this inequality and using $\sum_{i=0}^{2} \alpha_{i}=1$ yields

$$
\frac{\gamma_{0}\left(1+\gamma_{2}\right)-\gamma_{1}}{\gamma_{0}\left(\gamma_{1}-\gamma_{0}\right)}-\frac{\alpha_{2} \gamma_{1} \gamma_{2}}{\left(\gamma_{1}-\gamma_{0}\right)\left(1+\alpha_{2}\left(\gamma_{0}+\gamma_{1}\right)\right)} \geq 1-\alpha_{0},
$$

which requires $\frac{\gamma_{0}\left(1+\gamma_{2}\right)-\gamma_{1}}{\gamma_{0}\left(\gamma_{1}-\gamma_{0}\right)} \geq 1-\alpha_{0}$. If $\gamma_{1}>\gamma_{0}\left(1+\gamma_{2}\right)$, this inequality implies $\alpha_{0}>1$, which violates the constraint $\alpha_{0} \leq 1$ in (6e) and yields the statement of the lemma.

We will now use this lemma, to prove Theorem 1. Considering the equality in (8c), it can be seen from Lemma 2 that, because $\gamma_{1}>\gamma_{0}\left(1+\gamma_{2}\right)$, the coefficients of $\lambda_{1}$ and $\lambda_{3}$ in this equality are strictly positive. There are two distinct possibilities, namely, $\lambda_{1}=0$ and $\lambda_{1}>0$. 
Case $1\left(\lambda_{1}=0\right)$ : In this case, invoking (8a) yields $\lambda_{2}=1$. Substituting $\lambda_{1}=0$ and $\lambda_{2}=1$ in (8e) yields

$$
\frac{1+\gamma_{0}+\gamma_{2}+2 \sqrt{\alpha_{0} \beta_{0} \gamma_{0} \gamma_{2}}}{\left(1+\gamma^{\prime}\right)^{2}}=-\lambda_{3} \frac{\gamma_{2}\left(1-\beta_{0}\right)}{1+\gamma_{0}\left(1-\alpha_{0}\right)} \text {. }
$$

This equality is satisfied if and only if both sides of this equation are zero. Hence, $\gamma^{\prime}$ must be infinite and $\lambda_{3}=0$ or $\beta_{0}=1$.

We will show that $\lambda_{6}>0$. To do so, we consider (8b) and use $\lambda_{1}=0$ and $\lambda_{2}=1$ to write

$$
\sqrt{\gamma_{0} \gamma_{2}} \sqrt{\frac{\beta_{0}}{\alpha_{0}}}+\lambda_{3} \frac{\left(1-\beta_{0}\right) \gamma_{0} \gamma_{2} \gamma^{\prime}}{\left(1+\left(1-\alpha_{0}\right) \gamma_{0}\right)^{2}}+\lambda_{5}=\lambda_{6} .
$$

For any non-negative $\lambda_{3}$, if $0<\beta_{0} \leq 1$, the left hand side of (15) is strictly positive, which implies that $\lambda_{6}>0$. It remains to consider the case of $\beta_{0}=0$. In this case the first term on the left hand side of (15) vanishes and we are left with two possibilities: either $\lambda_{3} \gamma^{\prime}>0$, which yields $\lambda_{6}>0$, or $\lambda_{3} \gamma^{\prime}=0$. In the latter case, we consider (8d), from which we have

$$
\sqrt{\gamma_{0} \gamma_{2}} \sqrt{\frac{\alpha_{0}}{\beta_{0}}}+\lambda_{8}=\lambda_{7}
$$

Since $\beta_{0}=0$, we have from the last equality in (8f) with $i=7$ that $\lambda_{7}=0$. Using this observation and the fact that $\beta_{0}=0$ implies that $\sqrt{\frac{\alpha_{0}}{\beta_{0}}} \rightarrow \infty$ in (16) which is a contradiction ${ }^{1}$, i.e., the KKT system cannot be satisfied with $\lambda_{1}=0, \beta_{0}=0$ and $\lambda_{3} \gamma^{\prime}=0$.

Hence, for the KKT system in (8) to be satisfied with $\lambda_{1}=0$ when $\gamma_{1}>\gamma_{0}\left(1+\gamma_{2}\right)$, we must have $\lambda_{6}>0$. Using this in (8c) yields $\lambda_{4}>0$, which using (8f) yields the following optimal power partitions: $\alpha_{0}=1, \alpha_{1}=0, \alpha_{2}=0$. Using these partitions together with $\gamma^{\prime} \rightarrow \infty$, yields $R_{1}=0$ in (6c). Hence, the solution of the KKT system with $\lambda_{1}=0$ does not correspond to the maximum rate of the generalized DF-CF scheme.

Case $2\left(\lambda_{1}>0\right)$ : Using Lemma 2 , it can be seen that the first term in (8c) is strictly positive, which implies that $\lambda_{4}>0$ and thus $\alpha_{2}=0$. Substituting in (6c) yields

$$
R_{1}=\mathcal{C}\left(\alpha_{1} \gamma_{1}\right),
$$

which does not depend on $\beta_{0}$ and $\gamma^{\prime}$. Hence, choosing $\beta_{0}$ to maximize $R_{2}$ in (6d) does not reduce $R_{1}$. From (6d) it can be seen that $R_{2}$ is monotonically increasing in both $\beta_{0}$ and $\gamma^{\prime}$. However, from (6b), $\gamma^{\prime}$ is bounded by a monotonically increasing function of $\beta_{0}$. Hence, maximizing $\beta_{0}$ directly maximizes the positive term of $R_{2}$ and minimizes the negative term by maximizing the lower bound on $\gamma^{\prime}$. In particular, $R_{2}$ is maximized by setting $\beta_{0}=1$, which yields

$$
R_{2}=\mathcal{C}\left(\gamma_{0}+\gamma_{2}+2 \sqrt{\alpha_{0} \gamma_{0} \gamma_{2}}\right) .
$$

Hence, we have shown that the optimal solution of (7) corresponds to Case 2 in which $\alpha_{2}=0$ and $\beta_{1}=1-\beta_{0}=0$. Using these settings in Proposition 1 yields the statement of the theorem.

\footnotetext{
${ }^{1}$ Here we assume that $\sqrt{\frac{\beta_{0}}{\alpha_{0}}}=0$. Otherwise, $\lambda_{6}$ would be strictly positive as claimed.
}

\section{APPENDIX B}

\section{PROOF OF THEOREM 2}

To prove this theorem, we use the following two lemmas.

Lemma 3: For the Gaussian channel shown in Figure 1, the maximum rate that the generalized DF-CF scheme achieves using Gaussian signals is attained when the constraint in (6b) is satisfied with equality, that is, in that case,

$$
I\left(\hat{Y}_{1} ; Y_{1} \mid X_{2}, U, Y\right)=I\left(X_{2} ; Y \mid V\right) .
$$

Proof: We now prove the lemma using the KKT system (8). We note that another proof that uses a different approach has been obtained independently in Lemma 2 and Remark 5 of [27].

We consider the following three cases of $\alpha_{2}$ and $\left.\gamma^{\prime}: 1\right) \alpha_{2}=$ 0 , arbitrary $\gamma^{\prime}$; 2) $\alpha_{2}>0, \gamma^{\prime}<\infty$; and 3) $\alpha_{2}>0, \gamma^{\prime} \rightarrow \infty$. We will show that in each case if the maximum rate of the generalized DF-CF scheme is attained, the constraint on $\gamma^{\prime}$ in (6b) is satisfied with equality.

From the complementarity slackness conditions in (8f), it can be seen that if $\lambda_{3}$ were strictly positive, the constraint on $\gamma^{\prime}$ must be active and the lemma is automatically proved. Hence, to complete the proof of this lemma, it remains to consider the case of $\lambda_{3}=0$, which is what we do in the forthcoming analysis.

Case $1\left[\alpha_{2}=0\right.$ and $\gamma^{\prime} \geq 0$ ]: In this case, substituting in $(6 \mathrm{c})$ and $(6 \mathrm{~d})$ yields

$$
\begin{aligned}
& R_{1}=\mathcal{C}\left(\alpha_{1} \gamma_{1}\right), \quad \text { and } \\
& R_{2}=\frac{1}{2} \log _{2}\left(\left(1+\gamma_{0}+\gamma_{2}+2 \sqrt{\alpha_{0} \beta_{0} \gamma_{0} \gamma_{2}}\right) \frac{\gamma^{\prime}}{1+\gamma^{\prime}}\right) .
\end{aligned}
$$

Following an argument analogous to the one used in Case 2 in the proof of Theorem 1, it can be seen that in this case, the generalized DF-CF reduces to DF and $\gamma^{\prime} \rightarrow \infty$ which satisfies the constraint in (6b) with equality.

Case $2\left[\alpha_{2}>0\right.$ and $\left.\gamma^{\prime}<\infty\right]$ : Using $\lambda_{3}=0$ and that $\gamma^{\prime}$ is finite in (8e) and (8d) yields

$$
\lambda_{1} \frac{\alpha_{2} \gamma_{1}\left(1+\left(1-\alpha_{0}\right) \gamma_{1}\right)}{1+\alpha_{2} \gamma_{1}}=\lambda_{2}\left(1+\gamma_{0}+\gamma_{2}+2 \sqrt{\alpha_{0} \beta_{0} \gamma_{0} \gamma_{2}}\right)
$$

and

$$
\lambda_{2} \sqrt{\frac{\alpha_{0} \gamma_{0} \gamma_{2}}{\beta_{0}}} \frac{\gamma^{\prime}}{1+\gamma^{\prime}}+\lambda_{8}=\lambda_{7}
$$

Now, since, from (8a), $\lambda_{1}+\lambda_{2}=1$, it follows from (20) that, in this case, $\lambda_{1}>0$ and $\lambda_{2}>0$.

For $\gamma_{2}<\infty$, it can be seen from (6b) that $\gamma^{\prime}>0$. Using this observation in (21), we identify two possible cases: $\sqrt{\frac{\alpha_{0}}{\beta_{0}}}>0$ and $\sqrt{\frac{\alpha_{0}}{\beta_{0}}}=0$.

We will show that the first case yields a contradiction: Suppose that $\sqrt{\frac{\alpha_{0}}{\beta_{0}}}>0$. In this case, it is immediate that the left hand side of (21) is strictly positive and that $\lambda_{7}>0$. This implies that $\beta_{1}=0$ (cf. (8f)). Using this in (6b) yields $\gamma^{\prime} \geq \infty$, which contradicts the condition $\gamma^{\prime}<\infty$.

For the second case, we have $\sqrt{\frac{\alpha_{0}}{\beta_{0}}}=0$. Substituting for $\lambda_{3}=0$ in (8b) yields

$$
\lambda_{1}\left(1+\frac{\alpha_{2} \gamma_{1}}{1+\gamma^{\prime}}+\alpha_{2} \gamma_{0}\right) \frac{\gamma_{1}}{1+\alpha_{2} \gamma_{1}}+\lambda_{6}
$$




$$
=\lambda_{2} \sqrt{\frac{\beta_{0}}{\alpha_{0}}} \sqrt{\gamma_{0} \gamma_{2}} \frac{\gamma^{\prime}}{1+\gamma^{\prime}}+\lambda_{5} .
$$

Since $0<\lambda_{i}, \gamma_{j}, \gamma^{\prime}<\infty, i=1,2, j=0,1,2$, it can be seen that, because $\sqrt{\frac{\alpha_{0}}{\beta_{0}}}=0$, the right hand side of (22) is positive and infinite. Hence, it can be seen that $\lambda_{6}=\infty$. This implies that $\alpha_{0}+\alpha_{2}=1$. Invoking the fact that in this case $\alpha_{0}=0$, we have $\alpha_{2}=1$. Using this in (6c) and (6d) yields $R_{1}=\mathcal{C}\left(\gamma_{0}+\frac{\gamma_{1}}{1+\gamma^{\prime}}\right)$, and $R_{2}=\mathcal{C}\left(\gamma_{0}+\gamma_{2}\right)-\mathcal{C}\left(\frac{1}{\gamma^{\prime}}\right)$. From these expressions, we have $R_{1}$ monotonically increasing in $\gamma^{\prime}$ and $R_{2}$ monotonically decreasing in $\gamma^{\prime}$. Hence, maximizing $\min \left\{R_{1}, R_{2}\right\}$ is achieved by a value of $\gamma^{\prime}$ at which $R_{1}=R_{2}$. This equality, together with the particular value of $\gamma^{\prime}$ yield that in that case $\beta_{1}=1$ and (6b) holds with equality.

Case $3\left[\alpha_{2}>0\right.$ and $\left.\gamma^{\prime} \rightarrow \infty\right]$ : We consider two SNR regions: $\gamma_{0}<\gamma_{1}$ and $\gamma_{0} \geq \gamma_{1}$.

a) The case of $\gamma_{0}<\gamma_{1}$ : Using $\lambda_{3}=0$ and $\gamma^{\prime} \rightarrow \infty$ in (8c) yields

$$
\lambda_{1} \frac{\left(1+\left(1-\alpha_{0}\right) \gamma_{1}\right)\left(\gamma_{1}-\gamma_{0}\right)}{\left(1+\alpha_{2} \gamma_{1}\right)^{2}}-\lambda_{4}+\lambda_{6}=0 .
$$

When $\gamma_{0}<\gamma_{1}$, the coefficient of $\lambda_{1}$ is strictly positive. Following the proof of Theorem 1 it can be shown that the optimization leads to $\alpha_{2}=0$, which contradicts the condition $\alpha_{2}>0$.

b) The case of $\gamma_{0} \geq \gamma_{1}$ : In this case, we rewrite the rate expressions in Proposition 1 as

$$
\begin{aligned}
R_{1} & =\frac{1}{2} \log _{2} \frac{\left(1+\alpha_{2} \gamma_{0}\right)\left(1+\left(\alpha_{1}+\alpha_{2}\right) \gamma_{1}\right)}{1+\alpha_{2} \gamma_{1}}, \quad \text { and } \\
R_{2} & =\mathcal{C}\left(1+\gamma_{0}+\gamma_{2}+2 \sqrt{\alpha_{0} \beta_{0} \gamma_{0} \gamma_{2}}\right)
\end{aligned}
$$

Since $\gamma_{0} \geq \gamma_{1}$, then, from (23), $R_{1}$ is maximized when $\alpha_{2}=1$. However, in this case

$$
R_{1}=\mathcal{C}\left(\gamma_{0}\right)<\mathcal{C}\left(\gamma_{0}+\frac{\gamma_{1} \gamma_{2}}{1+\gamma_{0}+\gamma_{1}+\gamma_{2}}\right)=R_{C F}^{*}
$$

where the last equality follows from (10). Since $R_{G}^{*}$ is the maximum of $\min \left\{R_{1}, R_{2}\right\}$, (24) implies that $R_{G}^{*}$ is strictly less than the $\mathrm{CF}$ rate, which establishes the desired contradiction.

To conclude Case 3, we have, when $\gamma_{i}<\infty, i=0,1,2$, setting $\alpha_{2}>0$ and $\gamma^{\prime} \rightarrow \infty$ does not allow the generalized $\mathrm{DF}-\mathrm{CF}$ to attain its maximum rate.

Finally, gathering our results from Cases 1,2 and 3 and the case of $\lambda_{3}>0$, it can be seen that for all relevant instances of $\alpha_{2}$ and $\gamma^{\prime}$, the maximum rate that the generalized DF-CF scheme achieves using Gaussian signals is attained when the constraint in (6b) is satisfied with equality.

To complete the proof of Theorem 2, we need the following result.

Lemma 4: Rate $R_{1}$ and $R_{2}$ in Theorem 7 in [5] satisfy

$$
R_{1}=R_{2}+\Delta,
$$

where $\Delta=-I\left(X_{2}, U ; Y\right)+I\left(X_{2} ; Y \mid V\right)+I\left(U ; Y_{1} \mid X_{2}, V\right)$.

Proof: Invoking Lemma 3 in the expression of $R_{2}$ in Theorem 7 in [5] yields

$$
\begin{aligned}
R_{2} & =I\left(X_{1}, X_{2} ; Y\right)-I\left(\hat{Y}_{1} ; Y_{1} \mid X_{2}, X_{1}, U, Y\right) \\
& =I\left(X_{1}, X_{2} ; Y\right)-I\left(\hat{Y}_{1} ; Y_{1} \mid X_{2}, X_{1}, U, Y\right)
\end{aligned}
$$

$$
\begin{aligned}
& -I\left(X_{2} ; Y \mid V\right)+I\left(\hat{Y}_{1} ; Y_{1} \mid X_{2}, U, Y\right) \\
& =I\left(X_{1} ; Y \mid X_{2}\right)+I\left(X_{1} ; \hat{Y}_{1} \mid X_{2}, U, Y\right)+I(V ; Y) \\
& =I(V ; Y)+I\left(U ; Y \mid X_{2}, V\right)+I\left(X_{1} ; Y, \hat{Y}_{1} \mid X_{2}, U\right),
\end{aligned}
$$

where the second equality follows Lemma 3. The third and fourth equalities follows from using the chain rule and the fact that, by construction, $\left(V, X_{2}, Y\right)$ form the Markov chain $V \rightarrow X_{2} \rightarrow Y$ and that, conditional on $\left(X_{2}, U\right),\left(X_{1}, Y_{1}, \hat{Y}_{1}\right)$ form the Markov chain $X_{1} \rightarrow Y_{1} \rightarrow \hat{Y}_{1}$, and $\left(U, X_{1}, Y\right)$ form the Markov chain $U \rightarrow X_{1} \rightarrow Y$.

Substituting for $I\left(X_{1} ; Y, \hat{Y}_{1} \mid X_{2}, U\right)$ from (26) in the first term of $R_{1}$ in (3a) yields

$$
\begin{aligned}
R_{1} & =R_{2}-I(V ; Y)-I\left(U ; Y \mid X_{2}, V\right)+I\left(U ; Y_{1} \mid X_{2}, V\right) \\
& =R_{2}-I\left(X_{2}, U ; Y\right)+I\left(X_{2} ; Y \mid V\right)+I\left(U ; Y_{1} \mid X_{2}, V\right) \\
& =R_{2}-I\left(X_{2}, U ; Y\right)+I\left(X_{2} ; Y \mid V\right)+I\left(U ; Y_{1} \mid X_{2}, V\right) \\
& =R_{2}+\Delta,
\end{aligned}
$$

where $\Delta \triangleq-I\left(X_{2}, U ; Y\right)+I\left(X_{2} ; Y \mid V\right)+I\left(U ; Y_{1} \mid X_{2}, V\right)$. In writing (27) we have used the fact that, conditioned on $\left(X_{2}, U\right), Y$ is independent of $V$.

To proceed with the proof of Theorem 2, we invoke the construction in Section III-B into (25) to show that when $\gamma_{1} \leq$ $\gamma_{0}, \Delta \leq 0$; i.e., in that region $R_{1}$ is the constraining rate.

Invoking the construction in Section III-B, it is straightforward to show that

$$
\begin{aligned}
& I\left(X_{2}, U ; Y\right)=\frac{1}{2} \log _{2}\left(\frac{1+\gamma_{0}+\gamma_{2}+2 \sqrt{\alpha_{0} \beta_{0} \gamma_{0} \gamma_{2}}}{1+\alpha_{2} \gamma_{0}}\right), \\
& I\left(X_{2}, Y \mid V\right)=\frac{1}{2} \log _{2}\left(\frac{1+\left(\alpha_{1}+\alpha_{2}\right) \gamma_{0}+\beta_{1} \gamma_{2}}{1+\left(\alpha_{1}+\alpha_{2}\right) \gamma_{0}}\right), \\
& I\left(U, Y_{1} \mid X_{2}, V\right)=\frac{1}{2} \log _{2}\left(\frac{1+\left(\alpha_{1}+\alpha_{2}\right) \gamma_{1}}{1+\alpha_{2} \gamma_{1}}\right) .
\end{aligned}
$$

Substituting these expressions into the expression of $\Delta$ in Lemma 4 yields

$$
\begin{array}{r}
\Delta=\frac{1}{2} \log _{2}\left(\left(\frac{1+\left(\alpha_{1}+\alpha_{2}\right) \gamma_{1}}{1+\left(\alpha_{1}+\alpha_{2}\right) \gamma_{0}}\right)\left(\frac{1+\alpha_{2} \gamma_{0}}{1+\alpha_{2} \gamma_{1}}\right)\right. \\
\left.\left(\frac{1+\left(\alpha_{1}+\alpha_{2}\right) \gamma_{0}+\beta_{1} \gamma_{2}}{1+\gamma_{0}+\gamma_{2}+2 \sqrt{\alpha_{0} \beta_{0} \gamma_{0} \gamma_{2}}}\right)\right) .
\end{array}
$$

Noting that $\alpha_{1}+\alpha_{2} \leq 1$ and $\beta_{1} \leq 1$, it is immediate that $\frac{1+\left(\alpha_{1}+\alpha_{2}\right) \gamma_{0}+\beta_{1} \gamma_{2}}{1+\gamma_{0}+\gamma_{2}+2 \sqrt{\alpha_{0} \beta_{0} \gamma_{0} \gamma_{2}}} \leq 1$. Furthermore, it can be shown that, for $\gamma_{1} \leq \gamma_{0},\left(\frac{1+\left(\alpha_{1}+\alpha_{2}\right) \gamma_{1}}{1+\left(\alpha_{1}+\alpha_{2}\right) \gamma_{0}}\right)\left(\frac{1+\alpha_{2} \gamma_{0}}{1+\alpha_{2} \gamma_{1}}\right) \leq 1$, which implies that $\Delta \leq 0$. Using this in (25), we have that, when $\gamma_{1} \leq \gamma_{0}$, $R_{1}$ is the constraining rate and to maximize it we substitute for $R_{2}$ from (6d) and for $\Delta$ from (29) into (25), which yields

$$
\begin{aligned}
R_{1}=\frac{1}{2} \log _{2} & \left(\left(\frac{1+\left(\alpha_{1}+\alpha_{2}\right) \gamma_{1}}{1+\left(\alpha_{1}+\alpha_{2}\right) \gamma_{0}}\right)\left(\frac{1+\alpha_{2} \gamma_{0}}{1+\alpha_{2} \gamma_{1}}\right)\right. \\
& \left.\left(1+\gamma_{0}\left(\alpha_{1}+\alpha_{2}\right)+\gamma_{2} \beta_{1}\right)\left(\frac{\gamma^{\prime}}{1+\gamma^{\prime}}\right)\right) .
\end{aligned}
$$

Invoking Lemma 3 and substituting for $\gamma^{\prime}$ from (6b) in (30) yields

$$
R_{1}=\frac{1}{2} \log _{2}\left(\left(\frac{1+\left(\alpha_{1}+\alpha_{2}\right) \gamma_{1}}{1+\left(\alpha_{1}+\alpha_{2}\right) \gamma_{0}}\right)\left(\frac{1+\alpha_{2} \gamma_{0}}{1+\alpha_{2} \gamma_{1}}\right)\right)
$$




$$
\begin{gathered}
+\frac{1}{2} \log _{2}\left(\left(1+\alpha_{2}\left(\gamma_{0}+\gamma_{1}\right)\right)\left(1+\left(\alpha_{1}+\alpha_{2}\right) \gamma_{0}\right)\right) \\
+\frac{1}{2} \log _{2} \frac{\left(1+\left(\alpha_{1}+\alpha_{2}\right) \gamma_{0}\right)+\beta_{1} \gamma_{2}}{\beta_{1} \gamma_{2}\left(1+\alpha_{2} \gamma_{0}\right)+\left(1+\alpha_{2}\left(\gamma_{0}+\gamma_{1}\right)\right)\left(1+\left(\alpha_{1}+\alpha_{2}\right) \gamma_{0}\right)} .
\end{gathered}
$$

The first two terms are independent of $\left\{\beta_{j}\right\}_{j=0}^{1}$, and the last term is monotonically increasing in $\beta_{1}$. Hence, the optimal value of $\beta_{1}$ in this SNR range is 1 . Using this result, we rewrite $R_{1}$ as

$$
\begin{aligned}
R_{1}=\frac{1}{2} & \log _{2}\left(\left(\frac{1+\left(\alpha_{1}+\alpha_{2}\right) \gamma_{1}}{1+\left(\alpha_{1}+\alpha_{2}\right) \gamma_{0}}\right)\left(\frac{1+\alpha_{2} \gamma_{0}}{1+\alpha_{2} \gamma_{1}}\right)\right) \\
& +\frac{1}{2} \log _{2}\left(\left(1+\left(\alpha_{1}+\alpha_{2}\right) \gamma_{0}+\gamma_{2}\right)\right) \\
+ & \frac{1}{2} \log _{2}\left(\frac{1+\alpha_{2}\left(\gamma_{0}+\gamma_{1}\right)}{\frac{\gamma_{2}\left(1+\alpha_{2} \gamma_{0}\right)}{1+\left(\alpha_{1}+\alpha_{2}\right) \gamma_{0}}+\left(1+\alpha_{2}\left(\gamma_{0}+\gamma_{1}\right)\right)}\right) .
\end{aligned}
$$

Since $\gamma_{1} \leq \gamma_{0}$, it can be shown that the first term is upper bounded by 1 and the bound is reached when $\alpha_{1}=0$. The second term is maximized when $\alpha_{1}+\alpha_{2}=1$. For the last term, the denominator is minimized when $\alpha_{1}+\alpha_{2}=1$. The resulting expression is monotonically increasing in $\alpha_{2}$ and is maximized when $\alpha_{2}=1$. Hence, the rate maximizing power partitions for $\gamma_{1} \leq \gamma_{0}$ are $\alpha_{2}=1$ and $\beta_{1}=1$. From Proposition 1, such power partitions reduce the generalized DF-CF scheme to the CF scheme, which completes the proof of Theorem 2.

\section{APPENDIX C \\ PROOF OF LEMMA 1 AND COROLlary 1}

\section{A. Proof of Lemma 1}

The direct part is proved by substituting $\gamma_{1}\left(1+\gamma_{1}\right)=\gamma_{0}(1+$ $\left.\gamma_{0}+\gamma_{2}\right)$ in (10). Simplifying yields $R_{C F}^{*}=\mathcal{C}\left(\gamma_{1}\right)$. We will focus on the case of $\gamma_{2}>0$, i.e, the case in which the relaydestination link is not broken. In this case, $\gamma_{1}<\gamma_{0}+\gamma_{2}$. Using this in (9), yields $R_{D F, 1}<R_{D F, 2}$, which implies that $R_{D F}^{*}=R_{D F, 1}^{*}=\mathcal{C}\left(\gamma_{1}\right)$. This completes the proof of the direct part.

To prove the converse, we need to show that if $R_{D F}^{*}=R_{C F}^{*}$ then $\gamma_{1}\left(1+\gamma_{1}\right)=\gamma_{0}\left(1+\gamma_{0}+\gamma_{2}\right)$. We begin by providing an upper bound on the maximum achievable rate of the CF scheme using Gaussian signals, $R_{C F}^{*}$. From (10), we have, for any $\rho_{0} \geq 0$,

$$
\begin{aligned}
R_{C F}^{*} & =\mathcal{C}\left(\gamma_{0}+\frac{\gamma_{1} \gamma_{2}}{1+\gamma_{0}+\gamma_{1}+\gamma_{2}}\right) \\
& <\mathcal{C}\left(\gamma_{0}+\gamma_{2}+2 \sqrt{\rho_{0} \gamma_{0} \gamma_{2}}\right) .
\end{aligned}
$$

Comparing this bound with (9), it can be seen that $R_{C F}^{*}<$ $R_{D F, 2}$. This implies that if the maximum achievable rate of the DF scheme is given by $R_{D F}^{*}=R_{D F, 2} \leq R_{D F, 1}$, then $R_{C F}^{*}<$ $R_{D F}^{*}$. Hence for $R_{D F}^{*}$ to be equal to $R_{C F}^{*}, R_{D F, 1}$ must be the constraining rate of the DF scheme, i.e., $R_{D F}^{*}=R_{D F, 1}<$ $R_{D F, 2}$. However, from (9), this implies that $\gamma_{1}<\gamma_{0}+\gamma_{2}$. Otherwise, if this condition is not satisfied, the maximum rate of the DF scheme is achieved with $\rho_{i} \geq 0, i=0,1$, such that $\sum_{i=0}^{1} \rho_{i}=1$ and $R_{D F, 1}=R_{D F, 2}$.

From (9), it can be seen that, for $\gamma_{1}<\gamma_{0}+\gamma_{2}, R_{D F}^{*}=$ $R_{D F, 1}=\mathcal{C}\left(\gamma_{1}\right)$. Equating this rate with $R_{C F}^{*}$ in (10) yields $\gamma_{1}\left(1+\gamma_{1}\right)=\gamma_{0}\left(1+\gamma_{0}+\gamma_{2}\right)$, which completes the proof of the lemma.

\section{B. Proof of Corollary 1}

The SNR region $\gamma_{1}\left(1+\gamma_{1}\right)>\gamma_{0}\left(1+\gamma_{0}+\gamma_{2}\right)$ can be divided into two non-overlapping sub-regions: $\gamma_{1} \geq \gamma_{0}+\gamma_{2}$ and $\gamma_{0}+\gamma_{2}>\gamma_{1}>\frac{\gamma_{0}\left(1+\gamma_{0}+\gamma_{2}\right)}{1+\gamma_{1}}$.

For SNRs satisfying $\gamma_{1} \geq \gamma_{0}+\gamma_{2}$, it can be seen from (9) that $R_{D F}^{*}=R_{D F, 1}=R_{D F, 2}>\mathcal{C}\left(\gamma_{0}+\gamma_{2}\right)$. Now, the expression of $R_{C F}^{*}$ in (10) yields $R_{C F}^{*}<\mathcal{C}\left(\gamma_{0}+\gamma_{2}\right)$. Hence, in this SNR region, $R_{D F}^{*}>R_{C F}^{*}$.

We now consider the SNR region in which $\gamma_{0}+\gamma_{2}>$ $\gamma_{1}>\frac{\gamma_{0}\left(1+\gamma_{0}+\gamma_{2}\right)}{1+\gamma_{1}}$. In this region, we have from (9) that $R_{D F}^{*}=\mathcal{C}\left(\gamma_{1}\right)=R_{D F, 1}<R_{D F, 2}$. Using the fact that in this region $\gamma_{1}>\frac{\gamma_{0}\left(1+\gamma_{0}+\gamma_{2}\right)}{1+\gamma_{1}}$ in (10) yields $R_{D F}^{*}>R_{C F}^{*}$, which completes the proof of the first statement.

For the second statement of the corollary, it can be verified that the condition that $\gamma_{1}\left(1+\gamma_{1}\right)<\gamma_{0}\left(1+\gamma_{0}+\gamma_{2}\right)$ is equivalent to the condition that $\gamma_{1}<\gamma_{0}+\frac{\gamma_{1} \gamma_{2}}{1+\gamma_{0}+\gamma_{1}+\gamma_{2}}<\gamma_{0}+\gamma_{2}$. Using these inequalities to bound $R_{C F}^{*}$ in (10) and noting that in this region $R_{D F, 1}<R_{D F, 2}$, it follows that $R_{D F}^{*}<R_{C F}^{*}$, which completes the proof of the second statement.

\section{APPENDIX D \\ Proof of THEOREM 3}

To prove this theorem, we will use contradiction. First, we will assume that $R_{G}^{*}=R_{C F}^{*}=R_{D F}^{*}$, and then we will show that this $R_{G}^{*}$ and the corresponding power partitions and $\gamma^{\prime}$ do not satisfy the KKT necessary optimality conditions in (8).

First, we recall the result of Remark 1, indicating that the generalized DF-CF scheme reduces to the CF scheme when $\alpha_{2}=1$ and $\beta_{1}=1$. Substituting from (12) in (11) yields

$$
\gamma^{\prime}=\frac{\gamma_{0}}{\gamma_{1}-\gamma_{0}} \text {. }
$$

Note that the SNR condition in Lemma 1 implies that $\gamma_{1}>\gamma_{0}$, and hence that $0<\gamma^{\prime}<\infty$.

Next, we will examine the KKT system (8). We note that if $\alpha_{2}=1$ and $\beta_{1}=1$ are optimal, then for the complementary slackness equality in (8f) with $i=4,7$, we must have $\lambda_{4}=$ $\lambda_{7}=0$.

Substituting for $\gamma^{\prime}$ from (31) in (8c), the coefficient of $\lambda_{1}$ vanishes and using $\lambda_{4}=0$ yields $\lambda_{3} \frac{\gamma_{1}}{\left(1+\gamma_{0}\right)^{2}}+\lambda_{6}=0$, which implies that $\lambda_{3}=\lambda_{6}=0$. Using this result and the fact that $\gamma^{\prime}<\infty$ in (8e) yields $\lambda_{1} \gamma_{1}=\lambda_{2}\left(1+\gamma_{0}+\gamma_{2}\right)$.

Now, invoking $\lambda_{1}+\lambda_{2}=1$, yields $\lambda_{1}, \lambda_{2}>0$; both $\lambda_{1}$ and $\lambda_{2}$ are finite and bounded away from 0 . Substituting for $\lambda_{3}, \lambda_{7}$ and $\gamma^{\prime}$ in (8d) yields $-\lambda_{2} \sqrt{\frac{\alpha_{0}}{\beta_{0}}} \frac{\gamma_{0} \sqrt{\gamma_{0} \gamma_{2}}}{\gamma_{1}}=\lambda_{8}$. Since $\lambda_{2}>0$, for this equality to be satisfied, we must have $\lambda_{8}=0$, and

$$
\sqrt{\frac{\alpha_{0}}{\beta_{0}}}=0
$$

Substituting for $\lambda_{3}, \lambda_{6}$ and $\gamma^{\prime}$ in (8b) yields

$$
\lambda_{1} \gamma_{1}=\lambda_{2} \sqrt{\frac{\beta_{0}}{\alpha_{0}}} \frac{\gamma_{0} \sqrt{\gamma_{0} \gamma_{2}}}{\gamma_{1}}+\lambda_{5} .
$$

Now, the left hand side is finite. However, using (32), and the fact that $\lambda_{2}>0$ and $\lambda_{5} \geq 0$ implies that the right hand side is infinite. Hence, we conclude that the equality in (33) does not hold. This indicates that, when the SNRs satisfy the condition in Lemma 1 , setting $\alpha_{2}=1$ and $\beta_{1}=1$ does not satisfy the KKT conditions necessary for optimality. Hence, 
reducing the generalized DF-CF to the $\mathrm{CF}$ scheme by setting $\alpha_{2}=\beta_{1}=1$ is suboptimal; i.e., $R_{G}^{*}>R_{C F}^{*}$.

To complete the proof of the theorem, we note that at the SNRs satisfying Lemma $1, R_{D F}^{*}=R_{C F}^{*}$, which implies that, in this region, $R_{G}^{*}>R_{D F}^{*}$.

\section{APPENDIX E PROOF OF THEOREM 4}

Let $\Delta_{G}=R_{G}^{*}-\max \left\{R_{D F}^{*}, R_{C F}^{*}\right\}$ and consider the cases of $\gamma_{1} \leq \gamma_{0}$ and $\gamma_{1}>\gamma_{0}$.

From Theorem 2, when $\gamma_{1} \leq \gamma_{0}, R_{G}^{*}=R_{C F}^{*} \geq R_{D F}^{*}$, and hence in that region, $\Delta_{G}=0$.

We now consider the SNR region $\gamma_{1}>\gamma_{0}$. From the definition of $\Delta_{G}$, we can write

$$
\begin{aligned}
& \Delta_{G} \leq R_{G}^{*}-R_{D F}^{*} \\
& =\max _{\mathcal{S}} \min \left\{R_{1}, R_{2}\right\}-R_{D F}^{*} \\
& \leq \max _{\mathcal{S}} R_{1}-R_{D F}^{*},
\end{aligned}
$$

where $\mathcal{S}$ is the feasible set of the problem in (6), and $R_{1}$ and $R_{2}$ are defined in (6c) and (6d), respectively. Using (9) and the fact that $\rho_{i}^{*} \in[0,1], i=0,1$, and $\rho_{0}^{*}+\rho_{1}^{*}=1$, we have

$$
R_{D F}^{*}=\mathcal{C}\left(\gamma_{0}+\gamma_{2}+2 \sqrt{\rho_{0}^{*} \gamma_{0} \gamma_{2}}\right)=\mathcal{C}\left(\rho_{1}^{*} \gamma_{1}\right) .
$$

Using the monotonicity of the expression of $R_{2}$ in (6d) in $\gamma^{\prime}$, we can write

$$
R_{2} \leq \mathcal{C}\left(\gamma_{0}+\gamma_{2}+2 \sqrt{\alpha_{0} \beta_{0} \gamma_{0} \gamma_{2}}\right)
$$

Since $R_{G}^{*} \geq R_{D F}^{*}$, a comparison of (35) and (36) reveals that $\alpha_{0} \beta_{0} \geq \rho_{0}^{*}$. But, since $\beta_{0} \in[0,1]$, we must have $\alpha_{0} \geq \rho_{0}^{*}$. Hence, invoking $\sum_{i=0}^{2} \alpha_{i}=\sum_{i=0}^{1} \rho_{i}^{*}=1$ yields

$$
\alpha_{1}+\alpha_{2} \leq \rho_{1}^{*} \text {. }
$$

We will use (37) to obtain a bound on $\Delta_{G}$. In particular, from (34) and (35), we can write

$$
\begin{aligned}
\Delta_{G} & \leq \max _{\alpha_{1}, \alpha_{2} \in[0,1]} \frac{1}{2} \log \frac{\left(1+\alpha_{2}\left(\gamma_{0}+\gamma_{1}\right)\right)\left(1+\left(\alpha_{1}+\alpha_{2}\right) \gamma_{1}\right)}{\left(1+\alpha_{2} \gamma_{1}\right)\left(1+\rho_{1}^{*} \gamma_{1}\right)} \\
& \leq \max _{\alpha_{2} \in[0,1]} \frac{1}{2} \log \left(1+\frac{\alpha_{2} \gamma_{0}}{1+\alpha_{2} \gamma_{1}}\right) \\
& \leq \frac{1}{2} \log \left(1+\frac{\gamma_{0}}{1+\gamma_{1}}\right) \\
& <0.5 \mathrm{bpcu},
\end{aligned}
$$

where (38) follows from invoking (37), (39) follows from the monotonicity of the rational expression in $\alpha_{2}$, and the last inequality follows from using the fact that $\gamma_{1}>\gamma_{0}$.

\section{ACKNOWLEDGMENT}

The authors would like to thank Sekhar Chandra Bontu, Claude Royer, and Jim Womack of BlackBerry, formerly Research In Motion (RIM), for their support.

\section{REFERENCES}

[1] R. Pabst, B. H. Walke, D. C. Schultz, P. Herhold, H. Yanikomeroglu, S. Mukherjee, H. Viswanathan, M. Lott, W. Zirwas, M. Dohler, H. Aghvami, D. D. Falconer, and G. P. Fettweis, "Relay-based deployment concepts for wireless and mobile broadband radio," IEEE Commun. Mag., vol. 42, pp. 80-89, Sept. 2004.

[2] E. C. van der Meulen, "Three-terminal communication channels," Adv. Appl. Probab., vol. 3, pp. 120-154, 1971.

[3] T. M. Cover and J. A. Thomas, Elements of Information Theory. Wiley, 1991.

[4] T. J. Oechtering and H. Boche, "Bidirectional regenerative half-duplex relaying using relay selection," IEEE Trans. Wireless Commun., vol. 7, pp. 1879-1888, May 2008.

[5] T. M. Cover and A. A. El Gamal, "Capacity theorems for the relay channel," IEEE Trans. Inf. Theory, vol. IT-25, pp. 572-584, Sept. 1979.

[6] G. Kramer, M. Gastpar, and P. Gupta, "Cooperative strategies and capacity theorems for relay networks," IEEE Trans. Inf. Theory, vol. 51, pp. 3037-3063, Sept. 2005

[7] A. A. El Gamal, M. Mohseni, and S. Zahedi, "Bounds on capacity and minimum energy-per-bit for AWGN relay channels," IEEE Trans. Inf. Theory, vol. 52, pp. 1545-1561, Apr. 2006.

[8] X. Cai, Y. Yao, and G. B. Giannakis, "Achievable rates in low-power relay links over fading channels," IEEE Trans. Commun., vol. 53, pp. 184-194, Jan. 2005.

[9] W. Su and X. Lui, "Optimum selection relaying protocols in cooperative wireless networks," IEEE Trans. Commun., vol. 58, pp. 52-57, Jan. 2010.

[10] A. S. Avestimehr, S. N. Diggavi, and D. N. C. Tse, "Wireless network information flow: a deterministic approach," IEEE Trans. Inf. Theory, vol. 57, pp. 1872-1905, Apr. 2011.

[11] S. H. Lim, Y.-H. Kim, A. El Gamal, and S.-Y. Chung, "Noisy network coding," IEEE Trans. Inf. Theory, vol. 57, pp. 3132-3152, May 2011.

[12] L. Zhou and W. Yu, "Capacity of the Gaussian relay channel with correlated noises to within a constant gap," IEEE Commun. Lett., vol. 16, pp. 2-5, Jan. 2012.

[13] Y. Qi, R. Hoshyar, M. A. Imran, and R. Tafazolli, "H²-ARQ-relaying: spectrum and energy efficiency perspectives," IEEE J. Sel. Areas Commun., vol. 29, pp. 1547-1558, Sept. 2011.

[14] N. Ksairi, P. Ciblat, P. Bianchi, and W. Hachem, "Performance analysis over slow fading channels of a half-duplex single-relay protocol: decode or quantize and forward," IEEE Trans. Commun., vol. 60, pp. 20092016, July 2012.

[15] L. Zhang, J. Jiang, A. J. Goldsmith, and S. Cui, "Study of Gaussian relay channels with correlated noises," IEEE Trans. Commun., vol. 59, pp. 863-876, Mar. 2011.

[16] A. Høst-Madsen and J. Zhang, "Capacity bounds and power allocation for wireless relay channels," IEEE Trans. Inf. Theory, vol. 51, pp. 20202040, June 2005.

[17] B. Wang, J. Zhang, and A. Høst-Madsen, "On the capacity of MIMO relay channels," IEEE Trans. Inf. Theory, vol. 51, pp. 20-43, Jan. 2005.

[18] P. Razaghi and W. Yu, "Parity forwarding for multiple-relay networks," IEEE Trans. Inf. Theory, vol. 55, pp. 158-173, Jan. 2009.

[19] A. E. Gamal and M. Aref, "The capacity of the semideterministic relay channel," IEEE Trans. Inf. Theory, vol. 28, pp. 536-536, May 1982.

[20] C. Thomas M and Y.-H. Kim, "Capacity of a class of deterministic relay channles," Proc. 2007 IEEE Int. Symp. Inf. Theory, pp. 591-595.

[21] A. Høst-Madsen, "Deterministic capacity of networks," Proc. 2007 IEEE Inf. Theory Wkshp., pp. 601-606.

[22] J. Cui, "The capacity of Gaussian orthogonal multiple-access relay channel," IEEE Commun. Lett., vol. 15, pp. 365-367, Apr. 2011.

[23] A. S. Avestimehr, S. N. Diffavi, and D. N. C. Tse, "Approximate capacity of Gaussian relay netwoks," Proc. 2008 IEEE Int. Symp. Inf. Theory, pp. 474-478.

[24] P. Rost and G. Fettweis, "Analysis of a mixed strategy for multiple relay networks," IEEE Trans. Inf. Theory, vol. 55, pp. 174-189, Jan. 2009.

[25] S. R. Bhaskaran, "Forward decoding over a relay channel," Proc. 2008 IEEE Int. Symp. Inf. Theory, pp. 2673-2677.

[26] H.-F. Chong, M. Motani, and H. Krishna, "Generalized backward decoding strategies for the relay channel," IEEE Trans. Inf. Theory, vol. 53, pp. 394-401, Jan. 2007.

[27] H.-F. Chong and M. Motani, "On achievable rates for the general relay channel," IEEE Trans. Inf. Theory, vol. 57, pp. 1249-1266, Mar. 2011.

[28] C. W. Sung, Q. Wang, and K. W. Shum, "Capacity region of the linear four-node half-duplex wireless relay network," IEEE Commun. Lett., vol. 13, pp. 224-226, Apr. 2009.

[29] D. P. Bertsekas, Nonlinear Programming, 2nd edition. Athena Scientific, 1999. 


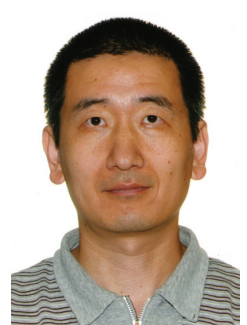

Kevin Luo received the B.Eng. degree from the Department of Information Engineering, Xidian University, Shaanxi, China in 1991, the M.Sc. degree from the Department of Electrical and Computer Engineering, University of Calgary, Alberta, Canada in 2004. He is currently pursuing the Ph.D. degree in the Department of Systems and Computer Engineering, Carleton University, Ottawa, Ontario, Canada.

Mr. Luo was a member of the Wireless Systems Group in Nortel Networks from 2004 to 2009, where he conducted research on the fourth-generation $(4 \mathrm{G})$

wireless communication systems.

His main research interests include information theory, signal processing and cross-layer design with their applications to cooperative communications in wireless networks.

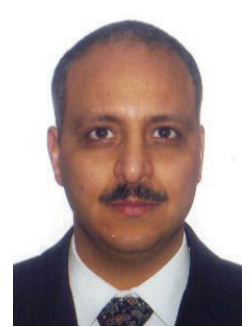

Ramy Gohary (S'02-M'06) received the B.Eng. (Hons.) degree from Assiut University, Egypt in 1996, the M.Sc. degree from Cairo University, Egypt, in 2000, and the Ph.D. degree from McMaster University, Ontario, Canada in 2006, all in electronics and communications engineering. $\mathrm{He}$ received the Natural Sciences and Engineering Research Council visiting fellowship award in 2007.

Dr. Gohary was a visiting scientist with the Terrestrial Wireless Systems Branch, Communications Research Centre, Canada. He is currently the project manager of the Carleton-BlackBerry (formerly Research in Motion (RIM)) research project.

His research interests include analysis and design of MIMO wireless communication systems, applications of optimization and geometry in signal processing and communications, information theoretic aspects of multiuser communication systems, and applications of iterative detection and decoding techniques in multiple antenna and multiuser systems.

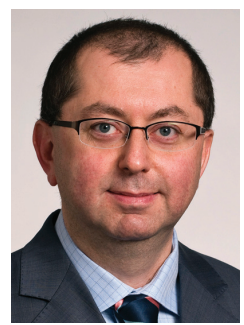

Halim Yanikomeroglu (S'96-M'98-SM'12) was born in Giresun, Turkey, in 1968. He received the B.Sc. degree in Electrical and Electronics Engineering from the Middle East Technical University, Ankara, Turkey, in 1990, and the M.A.Sc. degree in Electrical Engineering (now ECE) and the Ph.D. degree in Electrical and Computer Engineering from the University of Toronto, Canada, in 1992 and 1998, respectively. He was with the R\&D Group of Marconi Kominikasyon A.S., Ankara, Turkey, during 1993-1994.

Since 1998 Dr. Yanikomeroglu has been with the Department of Systems and Computer Engineering at Carleton University, Ottawa, where he is now a Full Professor. His research interests cover many aspects of wireless technologies with a special emphasis on cellular networks. Dr. Yanikomeroglu coauthored more than 50 journal and 160 conference papers, and has given more than 20 tutorials in leading international conferences on wireless technologies. In recent years, Dr. Yanikomeroglu's research has been funded by Huawei, BlackBerry (formerly Research in Motion (RIM)), Samsung, Communications Research Centre of Canada (CRC), Telus, and Nortel. This collaborative research resulted in about 15 patent applications.

Dr. Yanikomeroglu is a member of the Steering Committee of the IEEE Wireless Communications and Networking Conference (WCNC), and has been involved in the organization of this conference over the years, including serving as the Technical Program Co-Chair of WCNC 2004 and the Technical Program Chair of WCNC 2008; he will also serve as the Technical Program Co-Chair of WCNC 2014 to be held in Istanbul. Dr. Yanikomeroglu was the General Co-Chair of the IEEE Vehicular Technology Conference Fall 2010 held in Ottawa. Dr. Yanikomeroglu has served in the editorial boards of the IEEE Transactions on Communications, IEEE Transactions on Wireless Communications, and IEEE Communications Surveys \& Tutorials. He was the Chair of the IEEE Technical Committee on Personal Communications (now called, Wireless Technical Committee). He is a Distinguished Lecturer for the IEEE Vehicular Technology Society.

Dr. Yanikomeroglu is a recipient of the Carleton University Faculty Graduate Mentoring Award in 2010, the Carleton University Graduate Students Association Excellence Award in Graduate Teaching in 2010, and the Carleton University Research Achievement Award in 2009. Dr. Yanikomeroglu spent the 2011-2012 academic year at TOBB University of Economics and Technology, Ankara, Turkey, as a Visiting Professor. He is a registered Professional Engineer in the province of Ontario, Canada. 